\title{
Electronic structure of hcp transition metals
}

\author{
Jepsen, O.; Andersen, O. Krogh; Mackintosh, A. R.
}

Published in:

Physical Review B

Link to article, DOI:

10.1103/PhysRevB.12.3084

Publication date:

1975

Document Version

Publisher's PDF, also known as Version of record

Link back to DTU Orbit

Citation (APA):

Jepsen, O., Andersen, O. K., \& Mackintosh, A. R. (1975). Electronic structure of hcp transition metals. Physical Review B, 12(8), 3084-3103. https://doi.org/10.1103/PhysRevB.12.3084

\section{General rights}

Copyright and moral rights for the publications made accessible in the public portal are retained by the authors and/or other copyright owners and it is a condition of accessing publications that users recognise and abide by the legal requirements associated with these rights.

- Users may download and print one copy of any publication from the public portal for the purpose of private study or research.

- You may not further distribute the material or use it for any profit-making activity or commercial gain

- You may freely distribute the URL identifying the publication in the public portal

If you believe that this document breaches copyright please contact us providing details, and we will remove access to the work immediately and investigate your claim. 


\title{
Electronic structure of hep transition metals
}

\author{
O. Jepsen \\ AEK Research Establishment, Risø, Roskilde, Denmark \\ O. Krogh Andersen \\ Department of Electrophysics, Technical University, Lyngby, Denmark
}

A. R. Mackintosh

AEK Research Establishment, Risø, Roskilde, Denmark

and Rockwell Science Center, Thousand Oaks, California

(Received 14 April 1975)

\begin{abstract}
Using the linear muffin-tin-orbital method described in the previous paper, we have calculated the electronic structures of the hcp transition metals, $\mathrm{Zr}, \mathrm{Hf}, \mathrm{Ru}$, and Os. We show how the band structures of these metals may be synthesized from the $s p$ and $d$ bands, and illustrate the effects of hybridization, relativistic band shifts, and spin-orbit coupling by the example of Os. By making use of parameters derived from the muffin-tin potential, we discuss trends in the positions and widths of the energy bands, especially the $d$ bands, as a function of the location in the periodic table. The densities of states of the four metals are presented, and the calculated heat capacities compared with experiment. The Fermi surfaces of both Ru and Os are found to be in excellent quantitative agreement with de Haas-van Alphen measurements, indicating that the calculated $d$ band position is misplaced by less than $10 \mathrm{mRy}$. Very small pieces of Fermi surface, which have not yet been observed experimentally, are predicted for Os. The limited amount of experimental information available for $\mathrm{Zr}$ can be fairly satisfactorily interpreted if the calculated $d$ bands are raised by about 10-20 mRy relative to the $s p$ bands. This gives rise to a Fermi surface which is topologically equivalent to that recently found in $\mathrm{Ti}$, and which does not support open orbits when the magnetic field is sufficiently great that breakdown is complete. It is suggested that the Fermi surface of $\mathrm{Hf}$ is probably similar, although very little experimental evidence is available for this metal. Some comments are made about the calculational method, which has proved to be physically transparent, accurate and extremely fast, and the adequacy of the standard potential, which has now been successfully employed in calculations on the great majority of the transition metals.
\end{abstract}

\section{INTRODUCTION}

The hexagonal-close-packed structure is the most common among the transition metals, and the combination of the uniaxial symmetry and the two atoms per unit cell results in an electronic structure which is more complex than that of the cubic metals. This complexity is reflected in the Fermi surfaces, which have not generally been studied as intensively as those of more straightforward metals. However, a substantial amount of detailed experimental information is now available and the time seems ripe for an attempt at a quantitative interpretation of some of these experiments in terms of theoretical calculations of the band structure.

In this paper, we shall consider the $4 d$ and $5 d$ metals, in which relativistic effects are important, and report detailed calculations for $\mathrm{Zr}$ and $\mathrm{Hf}$ and for $\mathrm{Ru}$ and Os. These elements will serve as examples of the two groups, separated by the bcc metals in the periodic table, into which the hcp transition metals fall. A number of calculations have previously been performed on the $4 d$ and $5 d$ hcp metals. Loucks calculated the Fermi surfaces of $^{1} \mathrm{Y}$ and $\mathrm{d}^{2} \mathrm{Zr}$ using the nonrelativistic augmentedplane-wave (APW) method, while Keeton and Loucks ${ }^{3}$ included the relativistic effects in their study of Lu. The only case in which a detailed comparison is available between experiment and theory is $\mathrm{Re}$. Mattheiss ${ }^{4}$ calculated the electronic structure by the relativistic APW (RAPW) method, and his results are in generally good quantitative agreement with the de Haas-van Alphen (dHvA) measurements of Thorsen et al., ${ }^{5}$ the galvanomagnetic experiments of Reed et al., ${ }^{6}$ and the highfrequency magnetoacoustic results of Testarci and Soden, ${ }^{7}$ although there remain some questions about the small pieces of the Fermi surface. All of the above-mentioned calculations were based upon a non-self-consistent muffin-tin (MT) potential constructed from overlapping atomic charge densities, ${ }^{8}$ with the Slater $\rho^{1 / 3}$ approximation ${ }^{9}$ for the exchange and correlation. We have also used this potential, which has proved extremely successful in describing the electronic structure of the cubic transition metals, ${ }^{10-12}$ in our calculations. We have used relativistic Dirac-Slater atomic charge densities as our starting point throughout. Altmann and Bradley, ${ }^{13}$ on the other hand, used the cellular method for $\mathrm{Y}$ and $\mathrm{Zr}$, with a potential composed of a Hartree-Fock ionic term and a conduction-electron contribution approximated by a uniform charge distribution in the unit cell. Their results differ substantially from those of the APW calculations. 
As well as providing an interpretation of certain experimental results on the metals that we consider, and a prediction of other properties, this paper has a didactic purpose. We first show how the complicated band structures of the hcp metals are constructed from the relatively simple elements of the $s p$ and $d$ bands, emphasizing the characteristics of the latter, which are essential for an understanding of the properties of transition metals. For this purpose, we employ the atomic-sphere approximation (ASA) of Andersen, ${ }^{14}$ which is briefly discussed in Sec. II and described in detail in the preceding paper, ${ }^{15}$ hereafter referred to as I. In Sec. III we show the evolution of the band structure as the hybridization between the bands of different $l$ character is taken into account and the relativistic effects are included. In particular, we illustrate how the mass-velocity and Darwin terms give rise to quantitative shifts in the band positions, while the spin orbit coupling produces in addition qualitative changes in the connectivity of the bands. The trends in the band structures as a function of the position in the periodic table are discussed in Sec. IV, together with the details of the band structures and state densities of the four metals $\mathrm{Zr}, \mathrm{Hf}, \mathrm{Ru}$, and Os, calculated by means of the linear-muffintin-orbital (MTO) method, also presented in I. These band structures are used in Sec. V as the basis for an interpretation of the extensive dHvA measurements on $\mathrm{Ru},{ }^{16}$ and Os. ${ }^{17}$ Although the linear-MTO method has been checked by comparing the eigenvalues for hcp $\mathrm{Yb}$ with those derived from RAPW calculations, ${ }^{18}$ the calculations presented here provide the first published confrontation of the method with experimental evidence. As we shall see, the results are most satisfactory. Some comments are made on the limited amount of available experimental data on $\mathrm{Zr},{ }^{19}$ but, to our knowledge, no experiments pertaining directly to the Fermi surface of Hf have been performed. In conclusion, we summarize the results of our calculations and their implications, and suggest some experiments by which the electronic structures of the hcp transition metals might be further elucidated.

\section{METHOD OF CALCULATION}

The calculations presented in this paper were performed using the linear-MTO method which is derived in detail in I, and we shall be content here with a brief description of its concepts and principles. In addition, we will discuss a few practical details of the calculations.

In order to solve the band-structure problem for a MT potential, we define, for a single atomic sphere of radius $S$, the orbital

$$
\chi_{l m}(E, \overrightarrow{\mathrm{r}})=i^{l} Y_{l m}(\hat{r})
$$

$$
\times \begin{cases}\phi_{l}(E, r)+\frac{D_{l}(E)+l+1}{D_{l}(E)-l}(r / S)^{l}, & r \leq S \\ (r / S)^{-l-1}, & r \geq S .\end{cases}
$$

Here, $\phi_{l}$ is the solution at energy $E$ of the radial Schrödinger equation for the atomic-sphere potentail $v(r)$, which is the potential in the muffin-tin sphere, augmented by the constant $V_{\mathrm{mtz}}$ in the shell between the MT and atomic spheres. This orbital is continuous and differentiable provided that $D_{l}(E)$ is the logarithmic derivative function at the atomic sphere, i.e.,

$$
D_{l}(E) \equiv S \phi_{l}^{\prime}(E, S) / \phi_{l}(E, S) \text {. }
$$

For a crystal with lattice translations $\vec{R}$ and with equivalent atoms at positions $\vec{q}$ in the primitive cell, the linear combination

$$
\sum_{l m q} A_{l m q}^{\overrightarrow{\mathrm{k}}} \sum_{\overrightarrow{\mathrm{R}}} e^{i \overrightarrow{\mathrm{k}} \cdot \overrightarrow{\mathrm{R}}} \chi_{l m}(E, \overrightarrow{\mathrm{r}}-\overrightarrow{\mathrm{q}}-\overrightarrow{\mathrm{R}})
$$

is an approximate solution of the Schrödinger equation if, for the specified $E$ and $\overrightarrow{\mathrm{k}}$, the homogeneous equations

$$
\begin{aligned}
& \sum_{l m q}\left(-2(2 l+1) \frac{D_{l}(E)+l+1}{D_{l}(E)-l} \delta_{l^{\prime} l} \delta_{m^{\prime} m} \delta_{q^{\prime} \alpha}\right. \\
& \left.+S_{l^{\prime} m^{\prime} \alpha^{\prime} ; l m \alpha}^{\overrightarrow{\mathrm{k}}}\right) A_{l m \alpha}^{\overrightarrow{\mathrm{k}}}=0
\end{aligned}
$$

have a nontrivial solution. The approximation is that the atomic polyhedra may be simulated by spheres of the same volume and the condition for a solution is that, inside any atomic sphere, only the $\phi_{l}(E, r)$ terms of the corresponding orbitals survive, while the $(r / S)^{l}$ terms are canceled by the overlap from orbitals centered at the other sites of the crystal. Hence the Hermitian structure constants $s_{l^{\prime} m^{\prime} q^{\prime} ; l m q}^{\vec{a}}$ are multipole expansion coefficients, which are given explicitly in Paper I, Eq. (4.47).

The Eqs. (1) are those of Korringa, Kohn, and Rostoker, under the approximation that the interstitial region of the MT potential is taken into account by using the logarithmic derivatives at the atomic rather than at the MT sphere, and when $E-V_{\text {mtz }}$ is set equal to zero in the phase shifts and structure constants. This latter substitution has the important consequence that the structure constants become independent of energy and invariant under a uniform scaling of the crystal. These canonical structure constants may therefore conveniently be calculated once and for all throughout the irreducible Brillouin zones of the common crystal structures. Hence, the atomic-sphere approximation (ASA) makes possible a complete separation between the potential and energy dependen-cies, expressed in terms of the logrithmic derivative functions, and the dependence on the structure 
and wave vector, contained in the canonical structure constants. This separation may be extremely convenient, for example, in the consideration of the stability of different crystal structures as a function of electron concentration. ${ }^{20}$

We may diagonalize the $l l$ subblocks of the structure-constant matrix and obtain canonical bands $s_{l i}^{\vec{k}}$, and eigenvectors $U_{l m q}^{\vec{k}} ; l i$, independent of the potential and the atomic volume. If we then transform (1) to the $l i$ representation, the only off-diagonal matrix elements are the hybridization structure constants $\delta_{l^{\prime} i^{\prime}, l i}^{\vec{Z}}$, with $l \neq l^{\prime}$; the logarithmic derivative functions are invariant to this unitary transformation because they depend neither on $m$ nor $\vec{q}$, since all atomic-sphere potentials are equivalent and spherical. If the $l l^{\prime}$ hybridization is neglected, the band-structure problem in the ASA reduces to that of finding the eigenvalues for a single atomic sphere, subject to the $l_{-}, i_{-}$, and $\overrightarrow{\mathrm{k}}$-dependent boundary condition $D_{l}(E)=D_{l}^{\vec{k}}$, where

$$
D_{l i}^{\overrightarrow{\mathrm{k}}} \equiv-l-1-\frac{(2 l+1) s_{l i}^{\overrightarrow{\mathrm{k}}}}{2(2 l+1)-s_{l i}^{\overrightarrow{\mathrm{k}}}}
$$

or, equivalently, to finding the solutions of

$$
2(2 l+1) \frac{D_{l}(E)+l+1}{D_{l}(E)-l}=\mathrm{s}_{l i}^{\overrightarrow{\mathrm{s}}} .
$$

The logarithmic derivative is a decreasing function of energy, and the function on the left-hand side of (3) therefore increases with energy, diverging at the energies $E_{n l}(l)$ where $D_{l}=l$. The extent of the canonical band on the right-hand side of (3) is determined to a good approximation by the empirical Wigner-Seitz rule

$$
0 \geq D_{l i}^{\overrightarrow{\mathrm{k}}} \geq-\infty,
$$

so that the solutions $E_{n l}\left(D_{l i}^{\overrightarrow{\mathrm{k}}}\right)$ of (3), for a given $l$ but different values of $n$, lie in energy regions which are separated by the energies $E_{n l}(l)$. Moreover, the center of gravity of the canonical band is zero, so that

$$
\begin{aligned}
& \sum_{i} s_{l i}^{\overrightarrow{\mathbf{k}}}=0 \text { for all } \overrightarrow{\mathrm{k}} \text { and } l>0, \\
& \int s_{s}^{\overrightarrow{\mathbf{k}}} d^{3} k=0 \text { for } l=0 .
\end{aligned}
$$

Consequently, the canonical $l$ band $s_{l i}^{\overrightarrow{\mathrm{r}}}$ is transformed by the potential, or more specifically, the $l$ logarithmic derivative function, into unhybridized $n l$ bands $E_{n l}\left(D_{l i}^{\overrightarrow{\mathrm{k}}}\right)$ centered near $E_{n l}(-l-1)$ and extending approximately from $E_{n l}(0)$ to $E_{n l}(-\infty)$.

For each value of $l$, we are generally only interested in one of these bands, with $n=\nu$. For the $4 d$ transition metals, for example, we will consider the $4 d, 5 s$, and $5 p$ bands. In the vicinity of the energy of interest $E_{\nu}$, we may then parametrize the logarithmic derivative function so that we can solve
(3) explicitly, and write (1) in a form which is linear in energy, whereby the problem of hybridization can be solved by matrix-diagonalization techniques. This is accomplished by using, instead of the cancellation principle leading to (1), the variational principle for the Hamiltonian, together with trial functions which are linear combinations of energy-independent MTOs. The resulting linearMTO formalism is also very well.suited for the inclusion of corrections to the ASA, and spin-orbit coupling. In this formalism, we regard the logarithmic derivative, rather than the energy, as the independent variable, and in the atomic sphere, the radial part of the trial function of logarithmic derivative $D$ is the linear combination

$$
\Phi_{l}(D, r)=\phi_{l}\left(E_{\nu}, r\right)+\omega_{l}(D) \dot{\phi}_{l}\left(E_{\nu}, r\right)
$$

of the solution $\phi_{l}\left(E_{\nu}, r\right)$, normalized to unity in the sphere, and its energy derivative

$$
\ddot{\phi}_{l}\left(E_{\nu}, r\right) \equiv \frac{\partial \phi_{l}}{\partial E}
$$

The coefficient $\omega_{l}(D)$ may be expressed as

$$
\omega(D)=\omega(-l-1)+\frac{1}{2} S \Phi^{2}(-l-1, S)[s /(1-\gamma \delta)],
$$

where we have dropped the subscript $l$. The independent variable $D$ is related to $S$ as in (2), and

$$
\gamma \equiv \frac{1}{2(2 l+1)} \frac{\Phi(-l-1, S)}{\Phi(l, S)} .
$$

In the linear approximation, the inverse function of the logarithmic derivative function $D(E)$ is then

$$
\begin{aligned}
E(D) & =\frac{\left\langle\Phi(D)\left|-\nabla^{2}+v(r)\right| \Phi(D)\right\rangle}{\left\langle\Phi^{2}(D)\right\rangle} \\
& =E_{\nu}+\frac{\omega(D)}{1+\left\langle\dot{\phi}_{\nu}^{2}\right\rangle \omega^{2}(D)},
\end{aligned}
$$

and the potential parameters $\omega_{l}(-l-1), S \Phi_{l}^{2}(-l-1$, $S), \gamma_{l}$, and $\left\langle\dot{\phi}_{\nu l}^{2}\right\rangle$ can either be obtained by the fitting of (8) to the computed logarithmic derivative function or, according to (5), from the solution $\phi_{l}\left(E_{\nu}, r\right)$ and its energy derivative. The linear approximation is seen to be limited to the region of width $\left\langle\dot{\phi}_{\nu}^{2}\right\rangle^{-1 / 2}$ around $E_{\nu}$. If in (6) and (8) we use $D=D_{l i}^{\overrightarrow{\mathrm{k}}}$ and hence $\delta=\delta_{l i}^{\overrightarrow{\mathrm{k}}}$, we may conclude that the unhybridized energy-band structure is derived from the canonical band structure by fixing the band position through $E(-l-1) \approx E_{\nu}+\omega(-l-1)$, scaling it by

$\frac{1}{\mu S^{2}} \equiv-\left.\frac{1}{2} \frac{d E}{d D}\right|_{-l-1} \approx-\left.\frac{1}{2} \frac{d \omega}{d D}\right|_{-l-1}=\frac{1}{2} S \Phi^{2}(-l-1, S)$,

which is proportional to the probability amplitude at the atomic sphere, and distorting it nonlinearly by $\gamma$ and $\left\langle\dot{\phi}_{\nu}^{2}\right\rangle$. The values of the potential parameters for $\mathrm{Zr}$, $\mathrm{Hf}, \mathrm{Ru}$, and Os are given in Table I. 
TABLE I. Parameters from which the band structures of $\mathrm{Zr}, \mathrm{Hf}, \mathrm{Ru}$, and $\mathrm{Os}$ were calculated.

\begin{tabular}{|c|c|c|c|c|c|}
\hline & & $\mathrm{Zr}$ & Hf & $\mathrm{Ru}$ & Os \\
\hline Atomic number & & 40 & 72 & 44 & 76 \\
\hline Configuration & & $4 d^{3} 5 s^{1}$ & $5 d^{2} 6 s^{2}$ & $4 d^{7} 5 s^{1}$ & $5 d{ }^{6} 6 s^{2}$ \\
\hline Lattice constant (a.u.) & $\begin{array}{c}a \\
c / a\end{array}$ & $\begin{array}{l}6.1061 \\
1.5931\end{array}$ & $\begin{array}{l}6.0369 \\
1.5811\end{array}$ & $\begin{array}{l}5.1028 \\
1.5824\end{array}$ & $\begin{array}{l}5.1692 \\
1.5790\end{array}$ \\
\hline $\begin{array}{l}\text { Potential parameters } \\
E_{\nu}-V_{m t z}(\mathrm{Ry})\end{array}$ & & 0.600 & 0.600 & 0.900 & 0.900 \\
\hline$\omega(-l-1)(\mathrm{Ry})$ & $\begin{array}{l}s \\
p \\
d\end{array}$ & $\begin{array}{r}-0.133 \\
0.650 \\
0.118\end{array}$ & $\begin{array}{r}-0.236 \\
0.647 \\
0.168\end{array}$ & $\begin{array}{r}-0.249 \\
0.821 \\
-0.213\end{array}$ & $\begin{array}{r}-0.408 \\
0.798 \\
-0.159\end{array}$ \\
\hline $10 S \Phi^{2}(-l-1, S)$ & $\begin{array}{l}s \\
p \\
d\end{array}$ & $\begin{array}{l}2.606 \\
2.460 \\
0.514\end{array}$ & $\begin{array}{l}2.550 \\
2.508 \\
0.596\end{array}$ & $\begin{array}{l}3.754 \\
3.442 \\
0.566\end{array}$ & $\begin{array}{l}3.540 \\
3.392 \\
0.640\end{array}$ \\
\hline$\frac{\Phi(-l-1, S)}{\Phi(l, S)}$ & $\begin{array}{l}s \\
p \\
d\end{array}$ & $\begin{array}{l}0.854 \\
0.722 \\
0.161\end{array}$ & $\begin{array}{l}0.838 \\
0.714 \\
0.206\end{array}$ & $\begin{array}{r}0.851 \\
0.707 \\
-0.063\end{array}$ & $\begin{array}{l}0.836 \\
0.707 \\
0.021\end{array}$ \\
\hline$\left\langle\dot{\phi}_{\nu}^{2}\right\rangle^{-1 / 2}(\mathrm{Ry})$ & $\begin{array}{l}s \\
p \\
d\end{array}$ & $\begin{array}{l}3.4 \\
4.4 \\
1.08\end{array}$ & $\begin{array}{l}3.0 \\
4.6 \\
1.26\end{array}$ & $\begin{array}{l}4.7 \\
6.1 \\
1.07\end{array}$ & $\begin{array}{l}4.0 \\
5.1 \\
1.25\end{array}$ \\
\hline$\xi\left(D_{\nu}\right)(\mathrm{Ry})$ & $\begin{array}{l}p \\
d\end{array}$ & $\begin{array}{l}0.027 \\
0.0036\end{array}$ & $\begin{array}{l}0.094 \\
0.014\end{array}$ & $\begin{array}{l}0.045 \\
0.013\end{array}$ & $\begin{array}{l}0.150 \\
0.042\end{array}$ \\
\hline$\omega\left(D_{l}^{I}\right)(\mathrm{Ry})$ & $\begin{array}{l}p \\
d\end{array}$ & $\begin{array}{l}-21 . \\
-1.0\end{array}$ & $\begin{array}{l}-10 \\
-1.2\end{array}$ & $\begin{array}{l}-15 \\
-2.2\end{array}$ & $\begin{array}{l}-11 \\
-2.0\end{array}$ \\
\hline
\end{tabular}

The parameter $\mu$ introduced above is unity for free electrons of all $n l$ and $S$ [provided $D_{l}\left(E_{\nu}\right) \approx-l-1$ ], and since it is inversely proportional to the bandwidth, we name it the intrisic band mass. It is also illustrative to compare the relative band positions of crystalline and free electrons. For this purpose we use the parameter

$$
C_{l l^{\prime}} \equiv\left[E_{l}(-l-1)-E_{l^{\prime}}\left(-l^{\prime}-1\right)\right] S^{2},
$$

which for free $s p$ electrons takes the value $\frac{3}{4} \pi^{2}$ =7.40. If, at the center of the band, the kinetic energy is negative in the outer region of the atomic sphere, as is the case for the $d$ electrons in transition metals, then $\Phi^{2}(-l-1, S)$, and hence, the bandwidth and the distortion $\gamma$, are small. The $d$-band mass for the metals considered in Table II is around 4, and according to (4), (6), and (9), the $d$-band width is approximately $25 / \mu_{d} S^{2}$.

The linear-MTO matrix equivalent to (1) may be expressed as

$$
H_{l i ; l^{\prime}, i^{\prime}}^{\overrightarrow{\mathrm{k}}}-E O_{l i ; l^{\prime} i^{\prime},}^{\overrightarrow{\mathrm{k}}},
$$

where the formal one-center term of the Hamiltonian matrix, or rather $H-E_{\nu} O$, is

$$
\omega_{l}\left(D_{l i}^{\overrightarrow{\mathrm{x}}}\right) \delta_{l l^{\prime}}, \delta_{i i^{\prime}},
$$

and the formal one-center term of the overlap ma- trix is

$$
\left[1+\left\langle\dot{\phi}_{\nu l}^{2}\right\rangle \omega_{l}^{2}\left(D_{l i}^{\overrightarrow{\mathrm{k}}}\right)\right] \delta_{l l^{\prime}} \delta_{i i^{\prime}},
$$

The formal two- and three-center terms are, respectively, linear and quadratic in the hybridization matrix

$$
\begin{aligned}
T_{l i, l^{\prime} i^{\prime}}^{\overrightarrow{\mathrm{k}}} \equiv & \frac{\left(\sqrt{\frac{1}{2}} S\right) \Phi_{l}(-l-1, S)}{1-\gamma_{l} S_{l i}^{\overrightarrow{\mathrm{k}}}} \\
& \times s_{l i ; l^{\prime} i^{\prime}}^{\overrightarrow{\mathrm{k}}} \frac{\left(\sqrt{\frac{1}{2}} S\right) \Phi_{l^{\prime}}\left(-l^{\prime}-1, S\right)}{1-\gamma_{l^{\prime}} S_{l^{\prime} i^{\prime}}^{\overrightarrow{\mathrm{k}}}},
\end{aligned}
$$

which vanishes for $l^{\prime}=l$. The hybridization is thus proportional to the geometric mean of the bandwidths.

The relativistic effects originate in the regions close to the nuclei, where the electron velocity is high, and they can be neglected in the outer regions of the atomic spheres. When solving the relativistic band-structure problem, we may therefore formally use orbitals which are Pauli central-field spinors, but employing the logarithmic derivative functions

$$
D_{k}(E)=-(1+\kappa)+S c f_{k}(E, S) / g_{k}(E, S)
$$

derived from the radial Dirac equations. As usual, $\kappa$ is the combined quantum number for $j$ and $l^{10}$, and $c$ is the velocity of light. Furthermore, for 
TABLE II. Potential parameters for hep transition metals, calculated as described in the text. The values under Ru (NR) and Os (NR) were calculated with the same potential construction, but omitting the relativistic effects in the solid. The values under Os (NRA) were calculated with the omission of all relativistic effects, both in the atom and the solid. For comparison, the results of the free-electron (FE) model are included.

\begin{tabular}{lcccccc}
\hline \hline & $\mathrm{Y}$ & $\mathrm{Zr}$ & $\mathrm{Tc}$ & $\mathrm{Ru}$ & $\mathrm{Ru}(\mathrm{NR})$ & $\mathrm{FE}$ \\
\hline$S$ (a.u.) & 3.761 & 3.347 & 2.840 & 2.791 & 2.791 & \\
$C_{p s}$ & 8.52 & 8.61 & 8.38 & 8.21 & 8.02 & 7.40 \\
$C_{d s}$ & 4.07 & 2.79 & 0.74 & 0.34 & -0.20 & 17.72 \\
$\mu_{s}$ & 0.73 & 0.69 & 0.67 & 0.69 & 0.66 & 1 \\
$\mu_{p}$ & 0.81 & 0.77 & 0.77 & 0.79 & 0.76 & 1 \\
$\mu_{d}$ & 3.62 & 3.61 & 4.41 & 5.13 & 4.93 & 1 \\
$\xi_{d}$ (mRy) & 3.3 & 4.4 & 9 & 10 & 0 & 0 \\
& & & & & & \\
& $\mathrm{Lu}$ & $\mathrm{Hf}$ & $\mathrm{Re}$ & $\mathrm{Os}$ & $\mathrm{Os}$ (NR) & Os (NRA) \\
$S$ (a.u.) & 3.624 & 3.301 & 2.872 & 2.825 & 2.825 & 2.825 \\
$C_{p s}$ & 9.27 & 9.47 & 9.57 & 9.37 & 8.66 & 8.40 \\
$C_{d s}$ & 5.39 & 4.36 & 2.42 & 1.97 & 0.12 & -0.61 \\
$\mu_{s}$ & 0.76 & 0.73 & 0.71 & 0.73 & 0.64 & 0.65 \\
$\mu_{p}$ & 0.78 & 0.78 & 0.75 & 0.79 & 0.74 & 0.74 \\
$\mu_{d}$ & 3.28 & 3.25 & 3.71 & 4.11 & 3.68 & 4.40 \\
$\xi_{d}$ (mRy) & 14 & 18 & 31 & 35 & 0 & 0 \\
\hline \hline
\end{tabular}

the $4 d$ and $5 d$ metals, the spin-orbit separation between the energy functions inverse to (14) and corresponding to the same value of $l$

$$
E_{k=-l-1}(D)-E_{k=l}(D) \equiv\left(l+\frac{1}{2}\right) \xi_{l}(D)
$$

is much smaller than the bandwidths $E_{k}(\infty)-E_{k}(0)$, and the dependence of the spin-orbit coupling parameter in (15) on the logarithmic derivative is the same as that of the mass-velocity and Darwin band shifts, since it is a nonrelativistic normalization effect, which may be accounted for as follows. If

$$
\Phi\left(D^{I}, r\right)=\phi\left(E_{\nu}, r\right)+\omega\left(D^{I}\right) \dot{\phi}\left(E_{\nu}, r\right)
$$

is that linear combination which varies as $r^{l+2}$ near the nucleus, we may assume that matrix elements involving this function of any relativistic operator in the Pauli Hamiltonian vanish. The dependence of any relativistic shift or splitting on the logarithmic derivative is then simply obtained by using (16) to eliminate $\dot{\phi}$ in (5). For the spin-orbit coupling parameter we obtain

$$
\xi(D)=\frac{\left[1-\omega(D) / \omega\left(D^{I}\right)\right]^{2}}{1+\left\langle\dot{\phi}_{\nu}^{2}\right\rangle \omega^{2}(D)} \xi\left(D_{\nu}\right)
$$

The logarithmic derivative $D^{I}$ is easily computed as described in I, where it is also shown that the results of (15) and (17) are in good agreement for $\mathrm{Pt}$, the heaviest transition metal. Consequently, we shall use the Schrödinger representation with the orbitals $\chi_{l i}|\uparrow\rangle$ and $\chi_{l i}|\downarrow\rangle$, rather than the Pauli representation with its central-field spinors, and shall not therefore transform the canonical bands and hybridization structure constants. As described in I, the potential parameters $\omega_{l}(-l-1)$, $S \Phi_{l}^{2}(-l-1, S), \gamma_{l}$, and $\left\langle\dot{\phi}_{\nu l}^{2}\right\rangle$ are obtained by fitting to

$$
\left[(l+1) E_{k=-l-1}(D)+l E_{k=\imath}(D)\right] /(2 l+1) \equiv E_{l}(D)
$$

using the Dirac logarithmic derivatives, including the mass-velocity and Darwin shifts. Similarly, the two spin-orbit coupling parameters $\xi_{l}\left(D_{\nu l}\right)$ and $\omega_{l}\left(D_{l}^{I}\right)$ are obtained by fitting to (15), and we add the spin-orbit coupling matrix $[I,(5.7)]$ to the Hamiltonian matrix (11).

In the practical computations, we used the full linear-MTO formalism as described in I and, since the combined correction term and spin-orbit coupling were both included, we used the $l m q$ rather than the $l i$ representation, thus avoiding the $\overrightarrow{\mathrm{k}}-\mathrm{de}-$ pendent $U$ transformations. The latter procedure complicates the programming but speeds up the matrix diagonalization. In the hcp structure there are two atoms per unit cell and we included only $s, p$, and $d$ waves, so that $\max (l m q)=18$. The 50 structure constants [I, (4.48)] were computed by an Ewald technique, the 171 structure constants $[\mathrm{I},(4.17)]$ of the correction term were computed directly, and both sets of constants were stored on magnetic tape for 2541 points in the irreducible Brillouin zone. For the band structure shown in Figs. 3-9, the lattice parameters in Table I were used, but for the calculations of the state densities 
and Fermi surfaces a common $c / a$ ratio of 1.58 was adopted, in order that a single set of structure constants could be used. Test calculations for the lowest 10 bands at $90 \overrightarrow{\mathrm{k}}$ points showed that this procedure led to an rms error ranging from $0.3 \mathrm{mRy}$ for $\mathrm{Ru}$ to $2 \mathrm{mRy}$ for $\mathrm{Zr}$.

The potential was constructed by overlapping Dirac-Slater self-consistent relativistic atomic charge densities, which we calculated separately, using the Slater $\rho^{1 / 3}$ approximation for exchange and correlation, with $\alpha=1$. The potential parameters were obtained as described in I, by fitting to the Dirac logarithmic derivatives for the MT potential at the atomic sphere of radius $(3 \Omega / 8 \pi)^{1 / 3}$. The values of $E_{\nu}$ were chosen to be not too far from the estimated Fermi levels and the resulting parameters, from which our band structures may be reproduced, are shown in Table I. The eigenvalues were determined by a standard procedure involving Cholesky factorization of the overlap matrix and reduction of the transformed Hamiltonian to a real symmetric tridiagonal matrix. Even though this procedure is very efficient, it is the most timeconsuming factor in the calculations, and determines the speed of our method. With two atoms per unit cell and the inclusion of spin-orbit coupling, the matrices are complex and of dimension $36 \times 36$. With an IBM $360 / 75$ computer, all 18 Kramers-degenerate eigenvalues for a particular $\overrightarrow{\mathrm{k}}$ were obtained in about $1.5 \mathrm{sec}$. For the fcc and bcc structures, with one atom per unit cell, using the $l i$ representation and neglecting spin-orbit coupling, the nine eigenvalues per $\vec{k}$ point are obtained in about $0.1 \mathrm{sec}$, corresponding to approximately $10 \mathrm{msec}$ per eigenvalue.

The accuracy of the method has been discussed in I, where the empty-lattice test for free electrons is also considered. A few eigenvalues for the fcc transition metal Pd have been compared with APW calculations, ${ }^{14}$ but more relevant for the present application is a comparison between the linearMTO method and the relativistic APW method at $50 \overrightarrow{\mathrm{k}}$ points for the four lowest bands of hcp $\mathrm{Yb} .{ }^{18}$ It was found that the linear-MTO energies were on average $3 \mathrm{mRy}$ higher than the RAPW energies, which is in accord with the variational principle, that the rms fluctuation about this average was less than $3 \mathrm{mRy}$, and that the maximum deviation from the average was $+6 \mathrm{mRy}$. Considering that the use of the linear-MTO method in this case increased the computational speed by more than two orders of magnitude, this agreement must be considered very satisfactory.

The Fermi levels, state densities, and extremal Fermi-surface areas were computed automatically with a linear interpolation technique employing tetrahedral microzones. ${ }^{21}$ For $\mathrm{Ru}$, the eigenvalues were computed at 2541 points in the irreducible zone, which was therefore divided into 12000 tetrahedrons. This mesh is so fine that the error in all Fermi-surface areas due to the linear interpolation is negligible. In order to limit the use of computer time, we used only 396 points and 1500 tetrahedrons in the irreducible zone for the other metals. Comparing the results for the two meshes in $\mathrm{Ru}$, we conclude that the use of the coarse mesh may lead to errors of up to about $1 \mathrm{MG}$. This is negligible for large Fermi-surface areas, but may be significant for small sections. The use of the coarse mesh was as much dictated by the speed of the linear interpolation procedure as by the speed of the linear-MTO method. Hence, with this method, there is no incentive to use an intermediate interpolation scheme of the type frequently employed in connection with the APW and KKR methods.

\section{GENERAL FEATURES OF BAND STRUCTURES}

In this section, we shall consider how the band structure of an hcp transition metal is constructed from its constituent parts and illustrate the effects of hybridization, relativistic band shifts, and spinorbit coupling through the example of Os.

The Brillouin zone for the hexagonal structure is illustrated in Fig. 1. On account of the screw axis along [0001] in the hcp structure, all bands on the hexagonal face are at least fourfold degenerate (including spin), when spin-orbit coupling is neglected. It is therefore possible to adopt a double-zone representation, in which the number of bands is reduced by a factor of 2 , by allowing each to extend over twice the distance to the zone face in the hexagonal axis. However, spin-orbit coupling lifts this degeneracy, except along the line $R$, joining $A$ and $L$, and we shall therefore consistently use the single-zone representation, bearing in mind that magnetic breakdown may be important for $\vec{k}$-space orbits intersecting the hexagonal face of the zone, especially near the line $R$. The



FIG. 1. Brillouin zone for the hexagonal structure. 

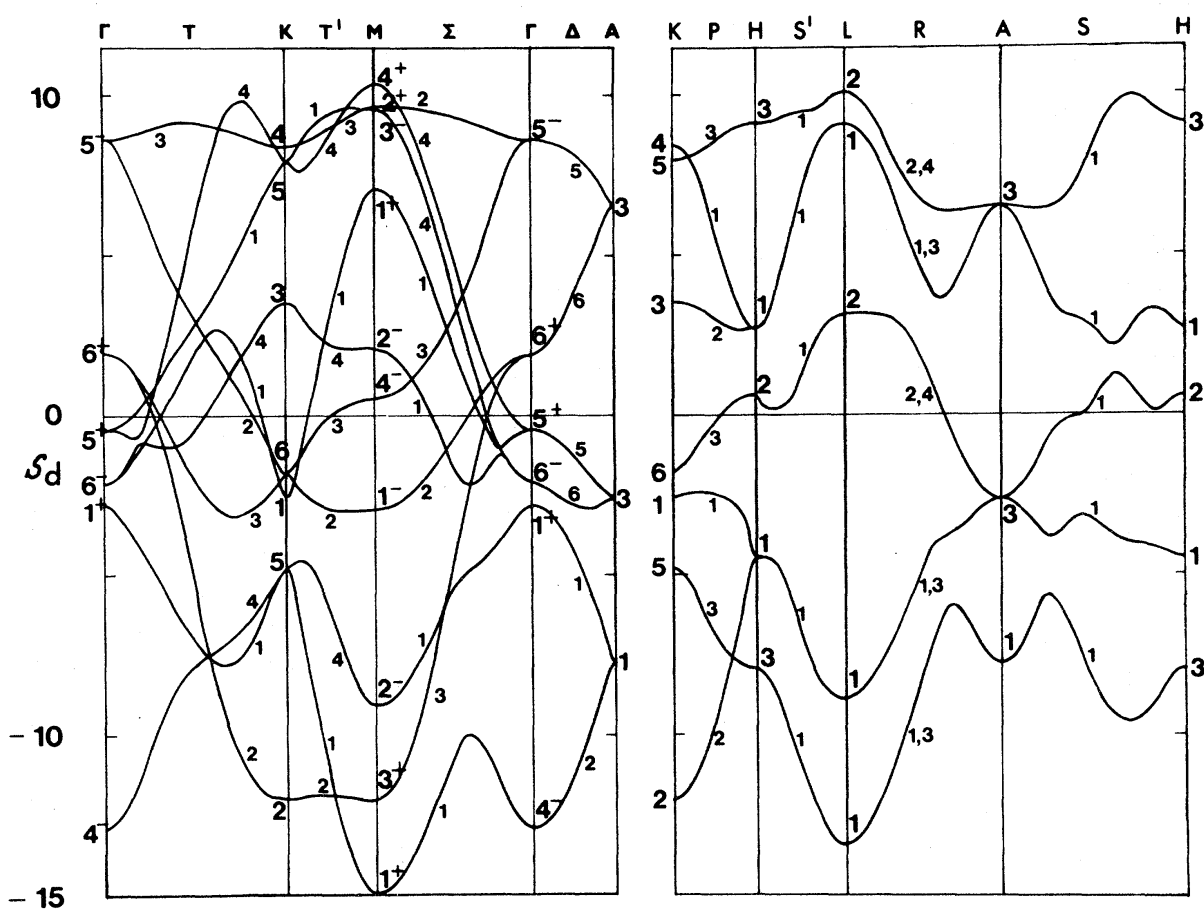

FIG. 2. Canonical $d$ bands $\delta_{d i}^{\mathrm{k}}$ for the hep structure with the ideal $c / a$ ratio.

basis of two atoms per unit cell ensures that all hcp metals are compensated, i.e., the total volume of the electron Fermi surfaces is equal to that of the hole surfaces.

The canonical $d$-band structure $\delta_{d i}^{\vec{k}}$ is shown in Fig. 2 along the symmetry lines of the zone, for the ideal $c / a$ ratio of 1.633 . The degeneracy on the hexagonal zone face is apparent in this figure. In general there are ten bands, derived from the five possible $m_{d}$ states of the two atoms per unit cell. In Fig. 3 we show the band structure of Os in the ASA, neglecting all relativistic effects, and also eliminating the hybridization between the $s p$ and $d$ bands by setting the nondiagonal structure constants involving $l=2$ equal to zero. The $d$ bands bear a strong resemblance to the canonical $d$ bands, located in energy by the potential parameter $C_{d s}$ of Table II, and scaled uniformly by the parameter $\mu_{d}$. The nonuniform distortion represented by $\gamma_{d}$ and $\left\langle\dot{\phi}_{\nu d}^{2}\right\rangle$ of Table I is small. The difference between the ideal $c / a$ ratio and the value 1.579 appropriate for Os results in some relative changes in the levels, e.g., the $\Gamma_{6-}$ and $\Gamma_{1+}$ levels are interchanged. The $s p$ bands are much broader than the $d$ bands and indeed are about $30 \%$ broader than those of the free-electron model, as may be seen from the mass parameters $\mu_{s}$ and $\mu_{p}$ of Table II.

The effects of hybridization on the band structure may be appreciated by comparing Figs. 3 and 4, since all nondiagonal structure constants are included in the latter. In addition, the effect of the correction term on the purely $d$-like levels may also be observed, since it is included in Fig. 4 and omitted in Fig. 3. We may distinguish between strong hybridization in which bands of the same symmetry, which cross in the absence of hybridization, mix with each other to produce energy gaps, and ueak hybridization in which bands of the same symmetry repel each other but do not cross. The drastic effect of strong hybridization is illustrated for the $s p$ bands by the thick lines in Fig. 4 . Except at certain symmetry points, all bands hybridize either strongly or weakly, in contrast to the cubic metals, in which bands of purely $d$ character exist along certain symmetry lines. The lowest-lying $\Gamma_{4-}, M_{1^{+}}$, and $L_{1} d$ states all hybridize with $s$ states, leaving $K_{2}$ as the lowest unhybridized $d$ level. $\Gamma_{5^{-}}, \Gamma_{6^{+}}, K_{4}, M_{1^{-}}$, and $M_{2^{+}}$are also pure $d$ states. $\Gamma_{3^{+}}$and the high-lying $\Gamma_{2^{-}}$are pure $p$ states, while all others are hybridized.

The relativistic effects on the band positions are illustrated by a comparison of Fig. 4 with Fig. 5, which shows the band structure of Os including the Darwin and mass-velocity shifts, together with the correction of the ASA described in I. The combined effect of the mass-velocity lowering and the Darwin raising of the center of the $s$ band produces a net lowering of $0.33 \mathrm{Ry}$ with respect to the fixed potential, which is consistent with the value found for Re by Mattheiss. ${ }^{4}$ The mass-velocity correction lowers the centers of the $p$ and $d$ bands by 0.25 and $0.10 \mathrm{Ry}$, respectively, while the Darwin term is negligible for nonzero $l$. The result, as may be seen from Table II, is that the $d$ bands rise 


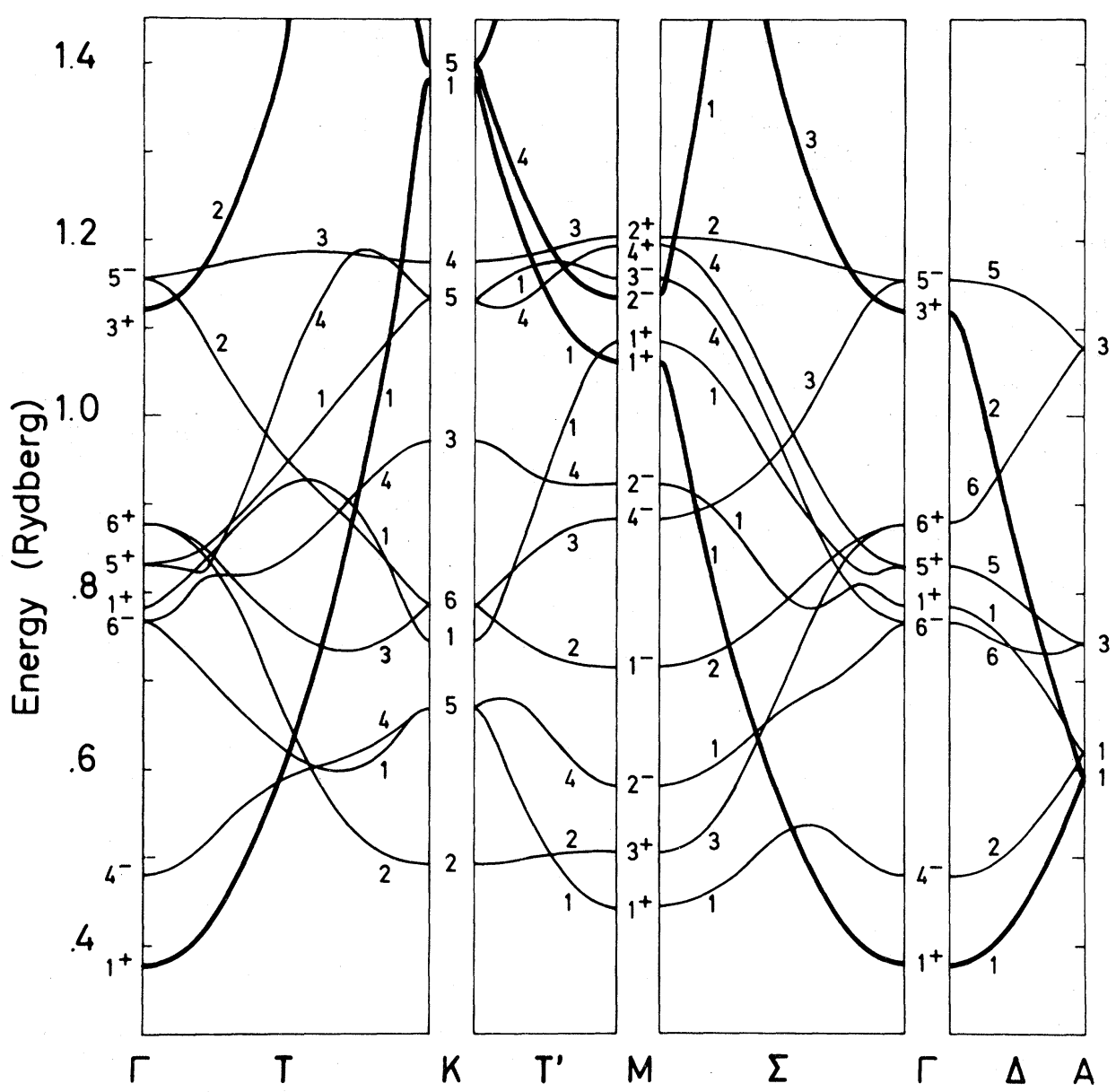

FIG. 3. Nonrelativistic band structure of Os with a $c / a$ ratio of 1.579 in the ASA, neglecting hybridization between the $s p$ bands, indicated by thick lines, and the $d$ bands.

relative to the $s p$ bands, and at the same time the normalization effect discussed in Sec. II causes all band masses to increase by roughly $10 \%$, leading to a relativistic narrowing of the bands.

Figure 6 is the same as Fig. 5, except that the spin-orbit coupling has been included by the method described in Sec. II. By altering the symmetry of the Hamiltonian, it has the effect of lifting the degeneracy on the hexagonal face of the Brillouin zone except along the line $R$ joining $A$ and $L$, and also of removing most of the accidental degeneracies along the symmetry lines. Band crossings remain possible in the $\Delta, P, S$, and $S^{\prime}$ directions, however. Because of the combined effects of timereversal symmetry and the center of symmetry in the hcp structure, all energy bands are at least doubly degenerate (including spin), even when spinorbit coupling is taken into account.

\section{POTENTIAL PARAMETERS, BAND STRUCTURES, AND STATE DENSITIES}

The most significant potential parameters for the $4 d$ and $5 d$ hcp transition metals are given in Table II. They are defined in (9), (10), and (17) with $D=-l-1$, and were calculated using the procedure outlined in Sec. II, with the lattice constants given by Pearson. ${ }^{22}$ The $5 s^{1}$ and $6 s^{2}$ configurations were used for the two periods. These were calculated to be the atomic ground states, and the difference may be attributed to the larger relativistic lowering of the $s$ level in the $5 d$ elements. The use of the $5 s^{2}$ and $6 s^{1}$ configurations for, respectively, $\mathrm{Zr}$ and Os changed $C_{d s}$ by, respectively, -32 and $+33 \mathrm{mRy}$.

The relative positions of the $s$ - and $p$-band centers are not too far from those of the free-electron model. For the $4 d$ metals $C_{p s}$ is about $15 \%$ larger, and for the $5 d$ about $25 \%$ larger than for free electrons. Similarly, the bandwidths are $(20-30) \%$ greater than those of the free-electron bands, and the masses $\mu_{s}$ and $\mu_{p}$ correspondingly lower. Although the $s p$ bands resemble a scaled version of the free-electron model, therefore, their precise form is difficult to reproduce with a weak local pseudopotential. The $d$ bands lie much lower than the free-electron $d$ bands, as is characteristic for transition metals. As the atomic number increases within a given period $C_{d s}$ decreases, on account of 




the increasingly negative effective potential experienced by the $d$ electrons, and for $\mathrm{Ru}$ the centers of the $s$ and $d$ bands lie very close. A con-

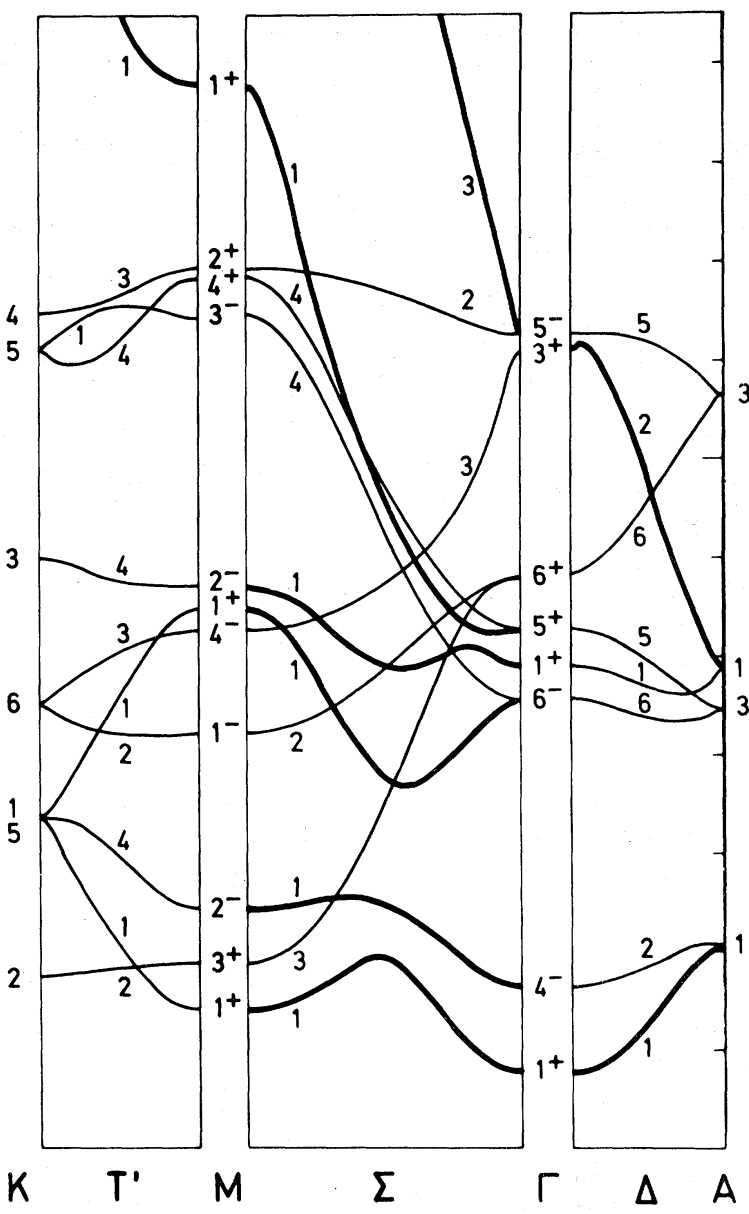

comitant increase occurs in $\mu_{d}$, so that the $d$ bands tend to narrow as they fall in energy. Because of the large relativistic lowering of the $6 s$ levels



FIG. 5. Relativistic band structure of Os neglecting spin-orbit coupling. The lowest-lying $\Gamma_{1+}$ state is essentially $s$ like, $\Gamma_{3+}$ is $p$ like, and $\Gamma_{5-}$ and $\Gamma_{6+}$ are purely $d$ like. 




FIG. 6. Relativistic band structure of Os, including spin-orbit coupling, calculated by the linear-MTO method.

mentioned earlier, the $5 d$ band lies relatively higher, and the center of this band is always substantially above that of the $6 s$ band. The $5 d$ masses are also somewhat smaller than the $4 d$. As may be seen by comparing the parameters for $\mathrm{Ru}$ and $\mathrm{Os}$, the relativistic band shifts in a particular column of the periodic table are roughly three times greater for the $5 d$ than for the $4 d$ metals. This is also true for the spin-orbit parameter $\xi_{\nu d}$, calculated at the center of the $d$ band, which increases rapidly with atomic number on account of the increasing effective nuclear charge near the nucleus, where relativistic effects are particulary important.

These band parameters are reflected in the electronic structures of the four metals $\mathrm{Zr}, \mathrm{Hf}, \mathrm{Ru}$, and Os, shown, respectively, in Figs. 7, 8, 9, and



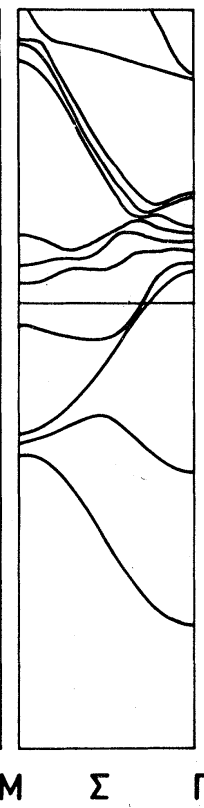

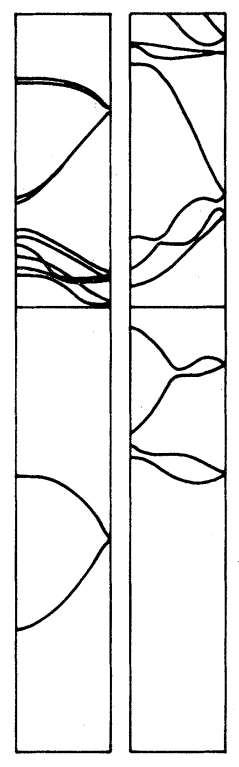

Г $\triangle A M U L K P H$
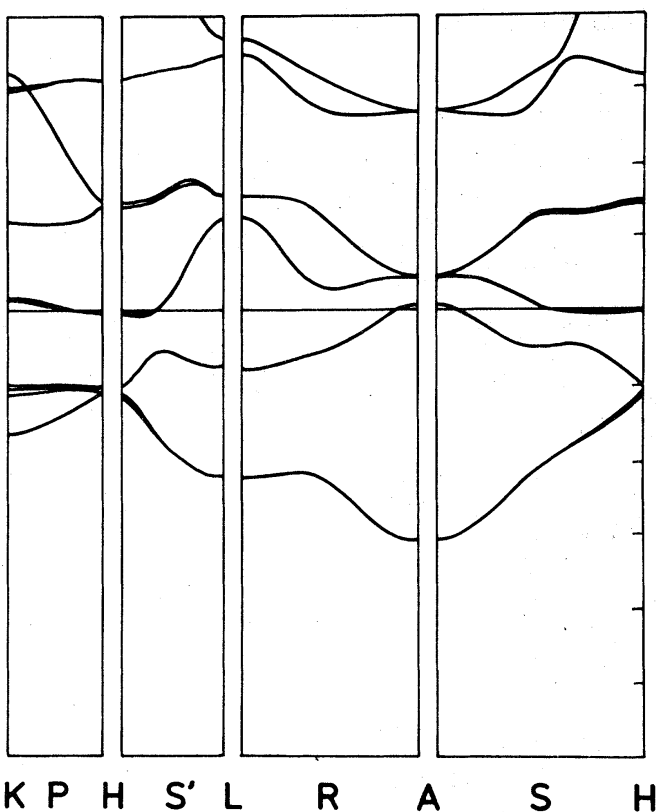

FIG. 7. Relativistic band structure of $\mathrm{Zr}$. 



FIG. 8. Relativistic band structure of Hf.

6 , which have been chosen as being characteristic of the two classes of hep transition metals. The state densities for these metals are shown, respectively, in Figs. 10, 11, 12, and 13. They are composed of a relatively small $s p$ contribution on which is superposed a large $d$ component with pronounced structure. As may be seen by comparing Figs. 13 with Fig. 14, which shows the state density of Os when all nondiagonal structure constants are neglected, hybridization between bands of different $l$ values has a profound effect on the structure in the density of states. Near the bottom of the $d$ band, strong hybridization between $s$ and $d$ states lowers the energy at which the rapid rise in the state density associated with the $d$ electrons occurs. At the top of the $d$ band, on the other


FIG. 9. Relativistic band structure of Ru. 


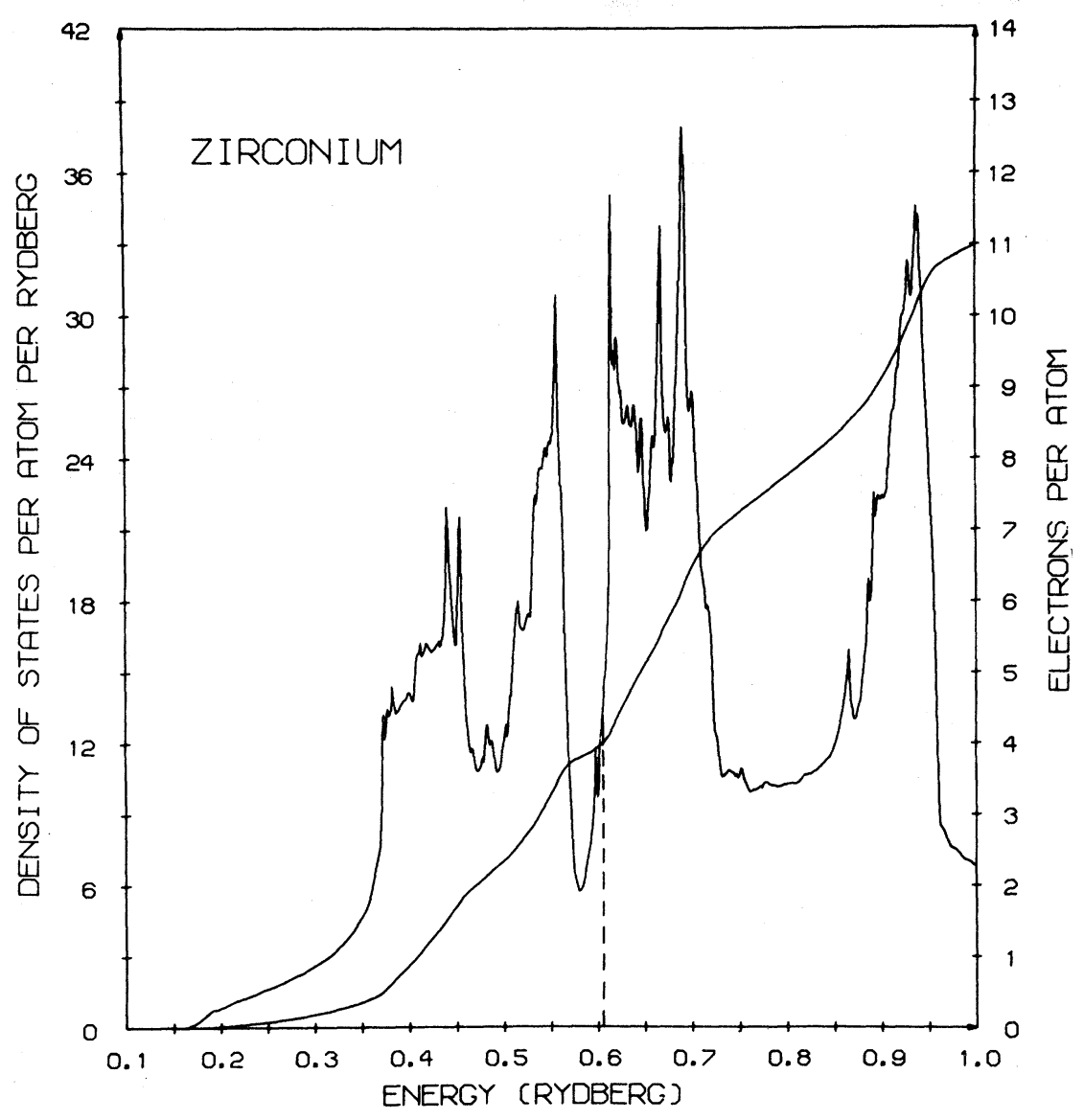

FIG. 10. Density of states of $\mathrm{Zr}$.

hand, the hybridization is weaker and, as we pointed out in Sec. III, many of the states are purely $d$-like, so that hybridization makes very little difference to the position of the final peak and the subsequent rapid drop in the state density. There are many pronounced peaks and dips in the density of states of Os which are not present in the canonical $d$ band state density, and these reflect the presence of hybridization energy gaps.

Although the $d$-band masses differ substantially in these metals, the $d$ band widths, which are inversely proportional to $\mu_{A} S^{2}$, i.e., approximately proportional to $S \Phi_{d}^{2}(-3, S)$, vary relatively little as may be seen in Table I. In Table III are given values of the Fermi surface parameter $s_{d}\left(E_{F}\right)$ calculated from the $d$ logarithmic derivative, from which the occupancy of the $d$ bands may be estimated by reference to Fig. 2. The approximate numbers of $d$ electrons $n_{d}$ are obtained from these parameters by integrating the canonical density of states up to $s_{d}\left(E_{F}\right)$, and are also given in the table. The state densities at the Fermi level $N\left(E_{F}\right)$ are given in Table III, together with the electronic heat capacities calculated from them. The measured values ${ }^{23}$ are also given and the ratio, which represents our estimate of the phonon enhancement of $N\left(E_{F}\right)$ is compared with the value deduced by McMillan ${ }^{24}$ from the superconducting properties. As may be seen in Table III, the agreement is satisfactory for $\mathrm{Ru}$ and Os but our calculations give a value of $N\left(E_{F}\right)$ which is somewhat too high, compared with McMillan's estimate, for $\mathrm{Zr}$ and Hf. An increase in the energy of the center of the $d$ band would result in a somewhat smaller increase in $E_{F}$, and therefore a relative decrease in the Fermi energy with respect to the rapid rise in $N(E)$ illustrated in Figs. 10 and 11 , with a corresponding decrease in $N\left(E_{F}\right)$. The heat-capacity results therefore indi-

TABLE III. Fermi-surface parameters and electronic heat capacities.

\begin{tabular}{lcccc}
\hline & $\mathrm{Zr}$ & $\mathrm{Hf}$ & $\mathrm{Ru}$ & $\mathrm{Os}$ \\
\hline$E_{F}-V_{\mathrm{mtz}}(\mathrm{Ry})$ & 0.605 & 0.622 & 0.827 & 0.898 \\
$\delta_{d}\left(E_{F}\right)$ & -4.76 & -5.46 & 5.10 & 4.86 \\
$n$ (states/atom) & 4 & 4 & 8 & 8 \\
$n_{d}($ states $/$ atom $)$ & 2.8 & 2.5 & 7.1 & 7.0 \\
$N\left(E_{F}\right)($ states $/$ atom $/$ Ry) & 13.1 & 10.9 & 11.8 & 9.2 \\
$\gamma_{\text {theory }}\left(\mathrm{mJ} / \mathrm{mole} \mathrm{K}^{2}\right)$ & 2.27 & 1.89 & 2.04 & 1.60 \\
$\gamma_{\text {expt }}\left(\mathrm{mJ} / \mathrm{mole}^{2}\right)^{\mathrm{a}}$ & 2.78 & 2.16 & 3.0 & 2.3 \\
$\gamma_{\text {expt }} / \gamma_{\text {theory }}$ & 1.22 & 1.14 & 1.47 & 1.44 \\
Theoretical enhancement $^{\mathrm{b}}$ & 1.41 & 1.34 & 1.38 & 1.39 \\
\hline \hline
\end{tabular}

${ }^{a}$ Ref. 23.
${ }^{\mathrm{b}}$ Ref. 24. 


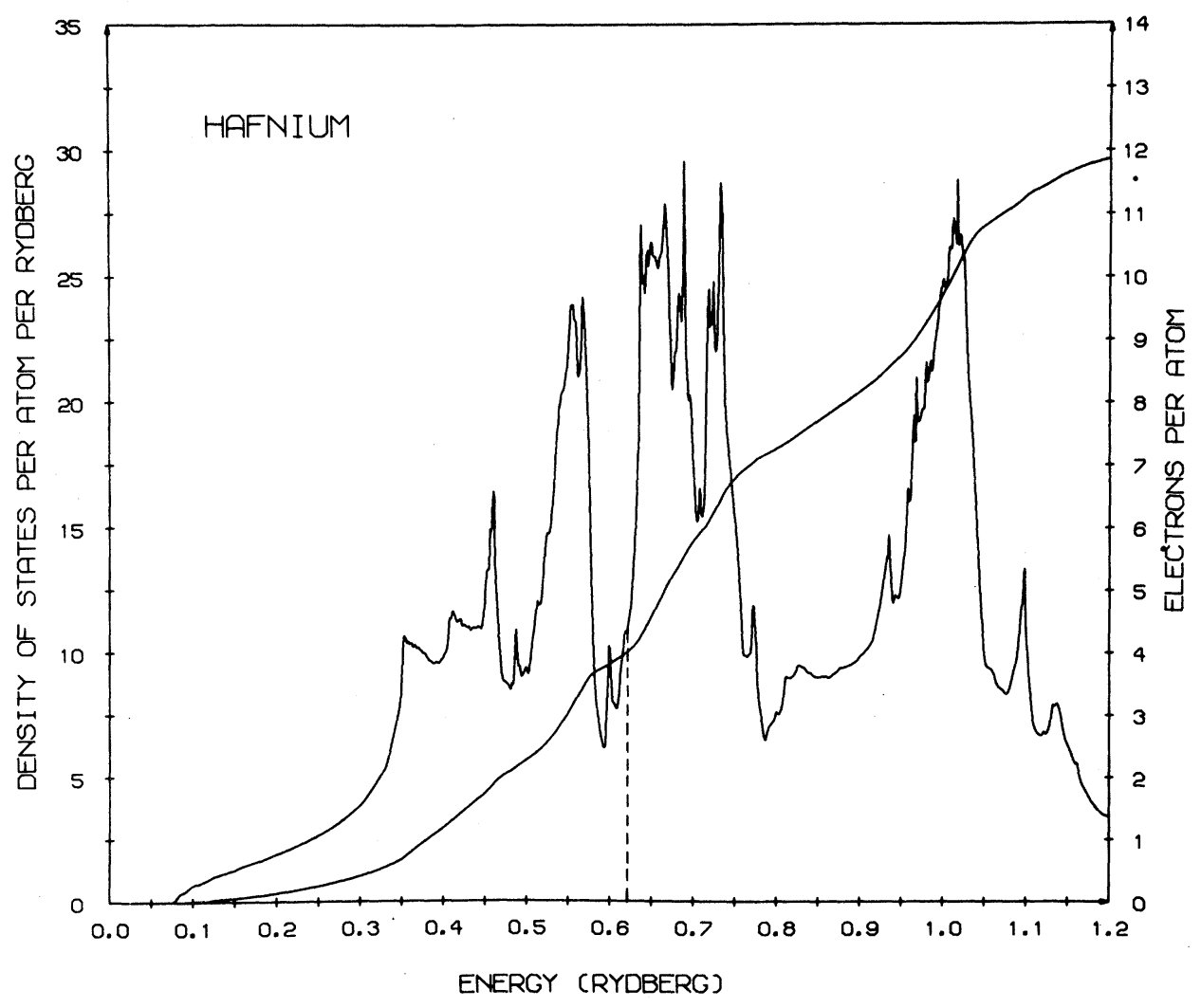

FIG. 11. Density of states of Hf.

cate that our calculated $d$ bands may lie somewhat too low in $\mathrm{Zr}$ and $\mathrm{Hf}$, and the significance of this observation for the Fermi surfaces will be discussed in Sec. V.

\section{FERMI SURFACES}

From the energy eigenvalues and the values of the Fermi energy $E_{F}$ given in Table III, we have calculated the Fermi surface sections shown in Figs. 15-18. Rather complete information on the Fermi surfaces of $\mathrm{Ru}$ and $\mathrm{Os}$ is available from dHvA measurements, and we shall first discuss these. According to our calculations, the two sets of surfaces are topologically equivalent. With a notation in which the position of the center of the surface (or the open direction of an open surface), the band in which it lies, and its electron or hole character are specified, each comprises one open $K M 8 h$ surface and five closed surfaces $\Gamma 9 e, \Gamma 10 e$, $U 7 h, L 7 h$, and $\Gamma 10 h$. The latter two are, however, very small in Os, so that a relative band shift of a few $\mathrm{mRy}$, which is within the combined uncertainty of the potential and calculational method, would eliminate them. We will discuss the evidence for their existence later in this section.

The Fermi surface of Ru has been studied in detail through the dHvA effect by Coleridge, ${ }^{16}$ who identified the observed orbits in terms of a Fermi

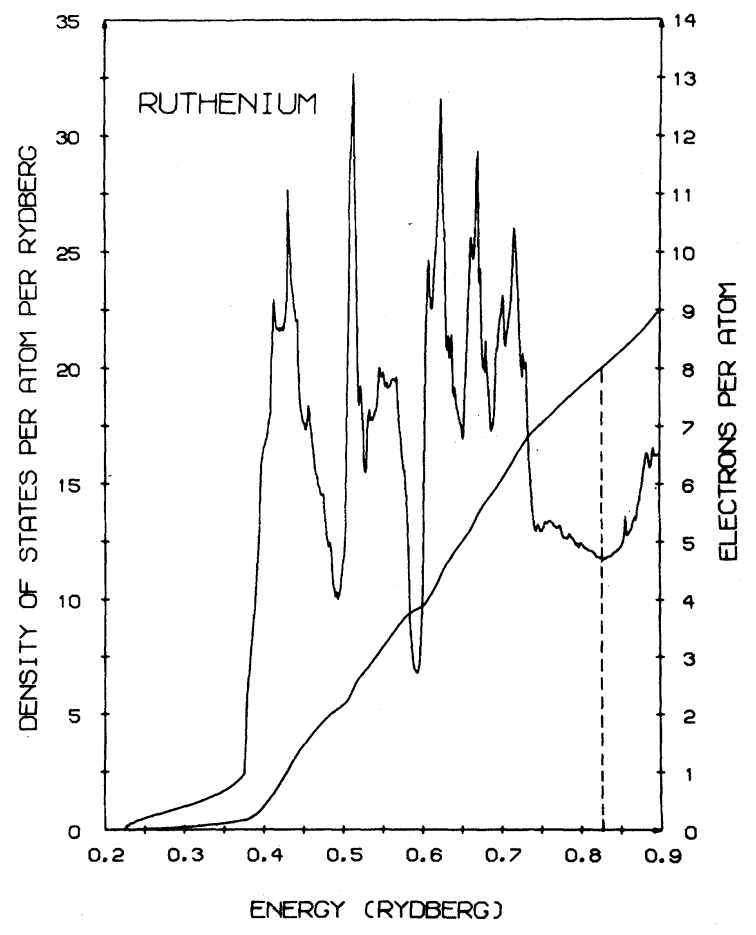

FIG. 12. Density of states of Ru. As explained in the text, these results were calculated with a finer mesh than was used for the other metals. 


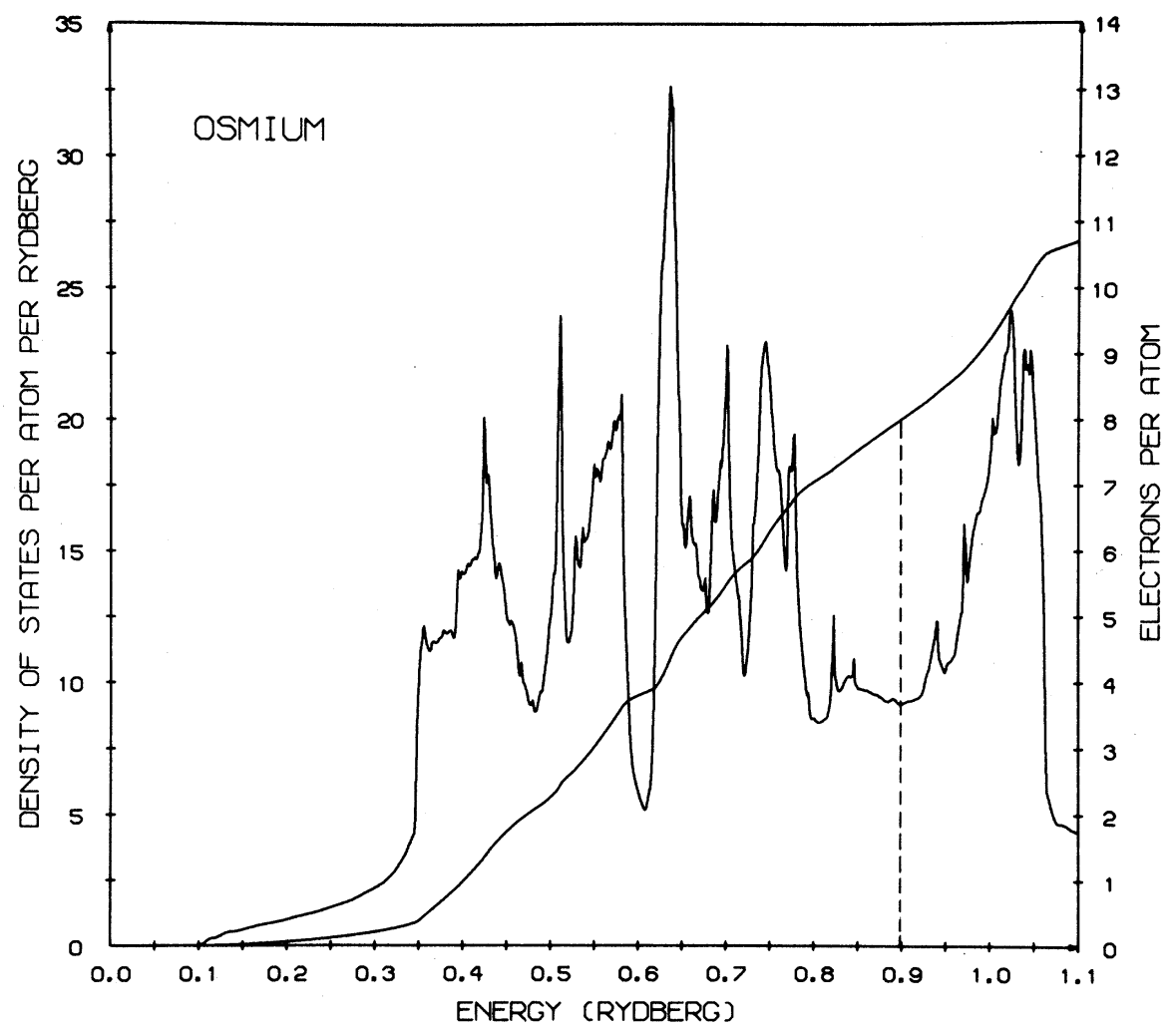

FIG. 13. Density of states of Os.

surface deduced from the nonrelativistic Re calculation of Mattheiss. ${ }^{4}$ His surface is qualitatively identical with that of Fig. 15, and we agree entirely with his interpretation, except that the magnitude of the observed area $\omega$ (see Table IV) indicates that it is associated with the orbit $\omega$ in Fig. 15 , rather than the somewhat smaller orbit $\omega^{\prime}$, even though the measured mass value appears to be anomalously low. As pointed out by Coleridge, the existence of magnetic breakdown on the hexagonal face of the Brillouin zone is essential for some of the observed orbits; for example, the orbits $\lambda$ which involve both the $K M 8 h$ and $L 7 h$ surfaces.

The agreement between our theoretical areas and the experimental results is excellent, to within a few percent for the large orbits, as may be seen from Table IV. Although the $L 7 h$ and $\Gamma 10 h$ surfaces are calculated to be somewhat larger than is observed experimentally, the over-all comparison between theory and experiment indicates that the bands near the Fermi level are correctly placed to within a few mRy. Of the smaller surfaces, $U 7 h$ is almost completely $d$ like, while the sizes of $\Gamma 10 h$ and $L 7 h$ are determined predominantly by the positions of the $p$-like $\Gamma_{3^{+}}$level and the $s p d-L_{1}$ level, respectively. Since the Fermi level in the transition metals is determined principally by the position of the $d$ bands, a shift in $C_{d s}$ is accom- panied by a shift in $E_{F}$ which is of the same sign and somewhat smaller. A raising of the $d$ band in $\mathrm{Ru}$ would therefore result in a small increase in the size of $U 7 h$, while both $\Gamma 10 h$ and $L 7 h$ would decrease by a relatively large amount. We therefore conclude that the $d$ band should be a few mRy higher than is indicated by our calculations. The masses on the large sheets of Fermi surface, which are predominantly $d$-like, are enhanced by an amount comparable with the phonon enhancement of the heat capacity, as expected, but the enhancement on $L 7 h$ and $\Gamma 10 h$, where the $d$ component is smaller, appears to be somewhat less.

Kamm and Anderson ${ }^{17}$ explained their dHvA results for Os by using the RAPW calculations on Re by Mattheiss, ${ }^{4}$ and again our interpretation is in essential agreement with theirs. As may be seen in Table IV, our calculations of the large orbit areas are in agreement with those observed experimentally, to within the uncertainty in the latter, and the enhancement of the masses is comparable with that of the electronic heat capacity. Principally because of the relativistic band shifts, we find that the small surfaces are even smaller than in $\mathrm{Ru}$, and the $\Gamma 10 h$ and $L 7 h$ surfaces have not been observed experimentally. However, galvanomagnetic measurements indicate that the $K M 8 h$ surface touches the hexagonal face of the Brillouin zone, which would imply the existence of $L 7 h$. 




FIG. 14. $s^{-}, p^{-}$, and $d$-state densities for Os. Relativistic potential parameters were used, but spin-orbit coupling was omitted.
The $U 7 h$ surface is calculated to be too large, and the use of a relatively coarse mesh for the Os calculations, although giving a negligible error for the larger surfaces, would tend to underestimate the true calculated areas on this small surface by as much as $20 \%$, implying that the discrepancy be- tween theory and experiment may be greater than indicated in Table IV. To bring the theory into agreement with experiment would require a lowering of the $d$ band by a few mRy, and this, would have the effect of increasing the sizes of $\Gamma 10 \mathrm{~h}$ and $L 7 h$, i. e., of making it more probable that they

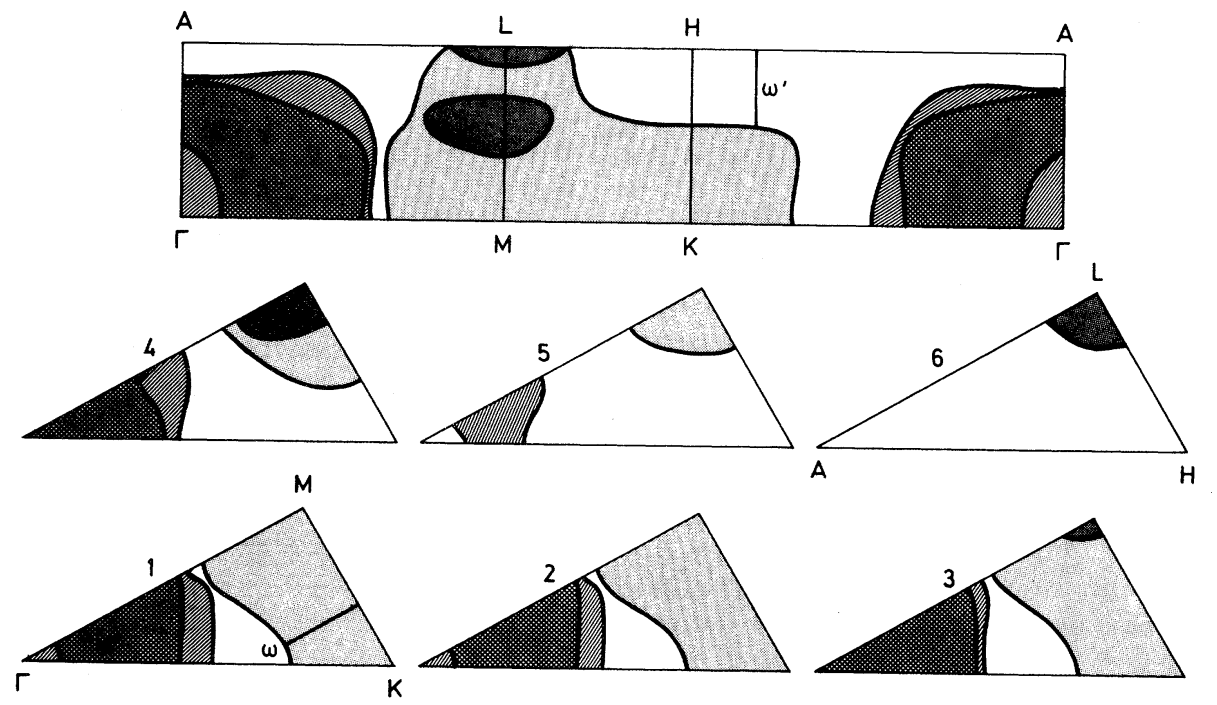

FIG. 15. Sections ot the Ru Fermi surface in the irreducible Brillouin zone. At the top are shown intersections with the faces of the irreducible zone, while six equidistant sections normal to [0001] are depicted at the bottom. 


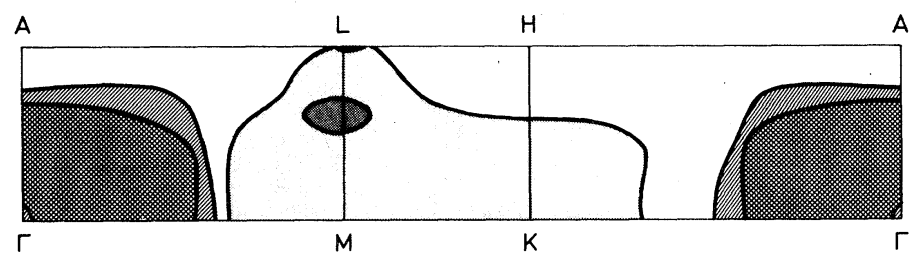

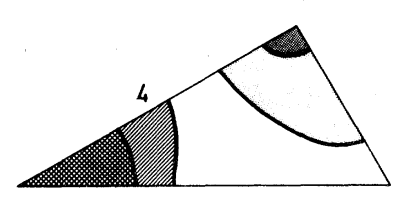

M
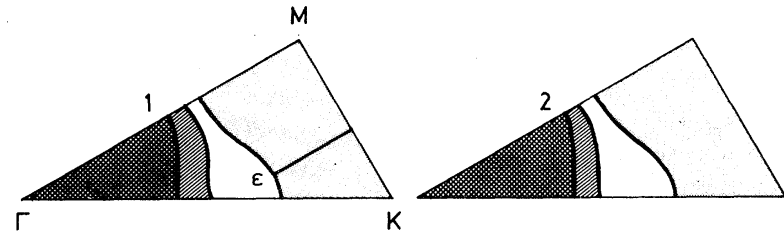

exist. Although it would take only very small relative shifts in our calculated bands to eliminate these surfaces, and these are unquestionably within the possible combined errors of the potential and calculations, the above considerations indicate that they may well exist, and it seems worthwhile to search for them with the dHvA effect. The example of ${ }^{25} \mathrm{Pd}$ demonstrates that very small pieces of Fermi surface may not always easily be detected with the dHvA effect.

Our band structure for $\mathrm{Zr}$ is rather similar to that of Loucks, ${ }^{2}$ but the inclusion of the relativistic band shifts has the effect of modifying the Fermi surface somewhat, most importantly in simplifying substantially the electron surfaces around $H$ (Fig. 17). At first sight, this Fermi surface does not appear to bear much relation to the relatively sparse experimental data and, in particular, there


FIG. 16. Sections of the Os Fermi surface in the irreducible Brillouin zone. is no sheet which can immediately account for the approximately isotropic $\alpha$ orbits observed by Everett. ${ }^{19}$ Further, we find that, contrary to his observations, the $\mathrm{Zr}$ Fermi surface is open in the [0001] direction, independently of whether magnetic breakdown occurs. A clue to these discrepancies may however be found in the very similar data on $\mathrm{Ti},{ }^{26}$ which have been satisfactorily explained by Jepsen. ${ }^{27}$ In $\mathrm{Ti}$, the $\Delta_{2}$ band intersects the Fermi level between $\Gamma$ and $A$, dissecting the $\Gamma A 3 h$ surface in the manner indicated by the dashed lines in Fig. 17. Following Loucks, ${ }^{2}$ we suggest that the same phenomenon occurs in $\mathrm{Zr}$. Since the $A_{1}$ level just above the Fermi level has $s p d$ character, this would require a relative raising of the $d$ bands by 10-20 mRy, with a concomitant increase in $E_{F}$ by a somewhat smaller amount. As mentioned in Sec. IV, such a shift seems to be indicated by the

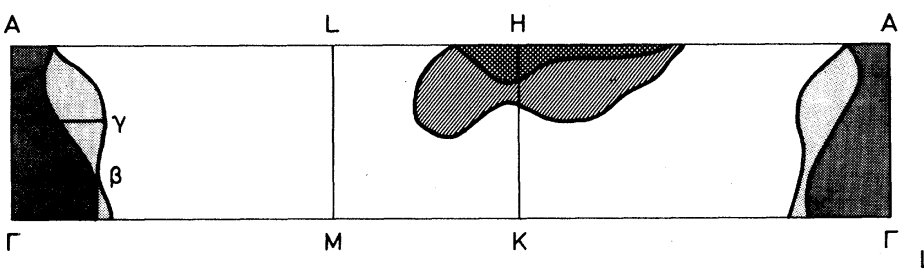



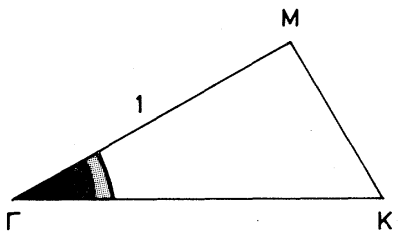



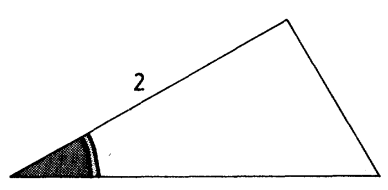

A
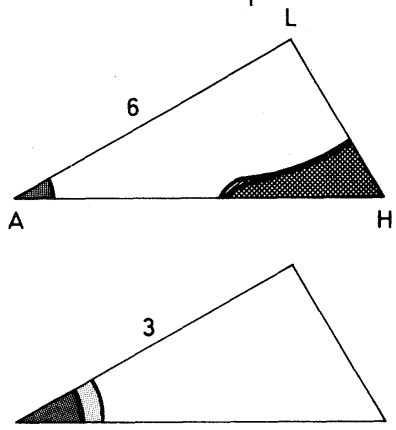

FIG. 17. Sections of the $\mathrm{Zn}$ Fermi surface in the irreducible Brillouin zone. 

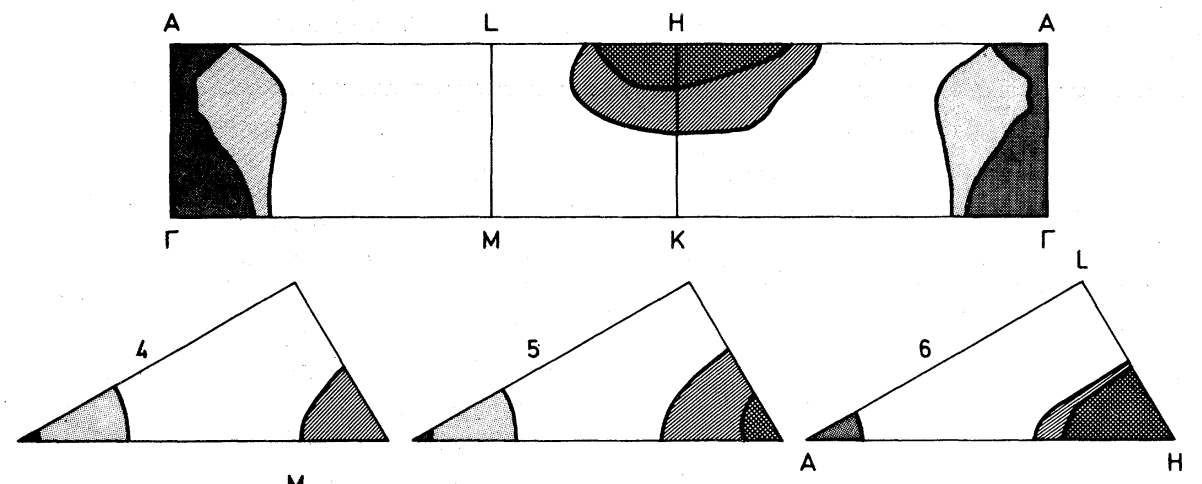

FIG. 18. Sections of the Hf Fermi surface in the irreducible Brillouin zone.
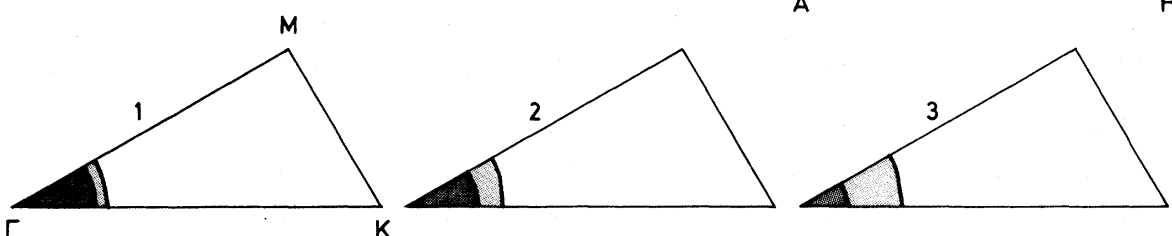

hence, as shown in Table $\mathrm{V}$, improve the agreement of the $\alpha, \beta$, and $\gamma$ orbits with experiment. Band shifts of 5-10 mRy relative to $E_{F}$ would bring these orbits into coincidence with the experi-

TABLE IV. Fermi-surface areas and cyclotron masses for Ru and Os. The experimental results for Ru are taken from Ref. 16 and those for Os from Ref. 17.

\begin{tabular}{|c|c|c|c|c|c|c|c|c|c|c|c|c|}
\hline \multirow{3}{*}{\multicolumn{2}{|c|}{ Direction }} & \multicolumn{5}{|c|}{ Ruthenium } & \multicolumn{6}{|c|}{ Osmium } \\
\hline & & \multicolumn{2}{|c|}{$\begin{array}{c}\text { Extremal areas } \\
\text { (MG) }\end{array}$} & \multicolumn{3}{|c|}{$\begin{array}{l}\text { Cyclotron masses } \\
\qquad\left(\mathrm{m} / \mathrm{m}_{0}\right)\end{array}$} & \multicolumn{3}{|c|}{$\begin{array}{l}\text { Extremal areas } \\
(\mathrm{MG})\end{array}$} & \multicolumn{3}{|c|}{$\begin{array}{l}\text { Cyclotron masses } \\
\qquad\left(\mathrm{m} / \mathrm{m}_{0}\right)\end{array}$} \\
\hline & & Theory & Expt. & Theory & Expt. & Ratio & & Theory & Expt. & Theory & Expt. & Ratio \\
\hline \multirow[t]{5}{*}{$L 7 h$} & [10 $\overline{1} 0]$ & 6.9 & & 0.29 & & & & 0.4 & & & & \\
\hline & {$[10 \overline{1} 0] \alpha$} & 5.6 & 3.5 & 0.21 & 0.24 & 1.1 & & 0.2 & & & & \\
\hline & {$[11 \overline{2} 0] \alpha$} & 6.1 & 4.5 & 0.25 & 0.26 & 1.1 & & 0.2 & & & & \\
\hline & {$[11 \overline{2} 0]$} & 5.7 & & 0.21 & & & & 0.2 & & & & \\
\hline & {$[0001] \alpha$} & 17.9 & 10.0 & 0.45 & 0.53 & 1.2 & & 0.7 & & & & \\
\hline \multirow[t]{5}{*}{$U 7 h$} & [10㐱0] & 10.8 & & 0.28 & & & $\alpha$ & 2.3 & 1.4 & 0.1 & 0.12 & \\
\hline & {$[10 \overline{1} 0] \beta$} & 7.5 & 7.5 & 0.20 & 0.27 & 1.4 & & 2.4 & & 0.1 & & \\
\hline & {$[11 \overline{2} 0] \beta$} & 13.4 & 13.0 & 0.35 & 0.43 & 1.2 & $\alpha$ & 2.1 & 1.5 & 0.1 & 0.14 & \\
\hline & {$[11 \overline{2} 0]$} & 8.2 & & 0.22 & & & $\alpha$ & 3.2 & 2.2 & 0.1 & 0.17 & \\
\hline & {$[0001] \beta$} & 22.1 & 20.5 & 0.38 & & & $\alpha$ & 5.7 & 2.9 & 0.2 & 0.22 & \\
\hline \multirow[t]{5}{*}{$K M 8 h$} & {$[10 \overline{1} 0] \lambda$} & 128.0 & 126.0 & 1.10 & 1.7 & 1.6 & $\delta$ & 111.0 & 110.0 & 1.00 & 1.49 & 1.5 \\
\hline & {$[11 \overline{2} 0] \lambda$} & 134.0 & 130.0 & 0.96 & 1.4 & 1.5 & $\delta$ & 109.0 & 109.0 & 0.87 & 1.10 & 1.3 \\
\hline & {$[11 \overline{2} 0] \omega$} & 61.1 & 60.5 & 1.16 & 1.2 & 1.0 & $\epsilon$ & 65.0 & 68.0 & 0.99 & & \\
\hline & {$[11 \overline{2} 0] \omega^{\prime}$} & 49.5 & & 0.77 & & & & 53.0 & & 0.92 & & \\
\hline & {$[0001]$} & 18.5 & & 0.42 & & & & 1.0 & & 0.31 & & \\
\hline \multirow[t]{3}{*}{$\Gamma 9 e$} & {$[10 \overline{1} 0] \sigma$} & 173.0 & 178.0 & 1.19 & & & $\gamma$ & 163.0 & 160.0 & 1.30 & & \\
\hline & {$[11 \overline{2} 0] \tau$} & 193.0 & 188.0 & 1.89 & & & $\gamma$ & 175.0 & 168.0 & 1.49 & & \\
\hline & {$[0001] \rho$} & 222.0 & 216.0 & 1.27 & 1.9 & 1.5 & $\gamma$ & 207.0 & 205.0 & 1.09 & & \\
\hline \multirow[t]{4}{*}{$\Gamma 10 e$} & {$[10 \overline{1} 0] \mu$} & 143.0 & 144.0 & 1.35 & 1.9 & 1.4 & $\beta$ & 124.0 & 124.0 & 1.12 & 1.2 & 1.1 \\
\hline & {$[11 \overline{2} 0] \mu$} & 153.0 & 153.0 & 1.45 & & & $\beta$ & 133.0 & 133.0 & 1.23 & 1.2 & 1.0 \\
\hline & {$[0001] \nu$} & 160.0 & 158.0 & 1.02 & 1.5 & 1.5 & $\beta$ & 152.0 & 153.0 & 0.97 & 1.2 & 1.2 \\
\hline & {$[0001] \nu$} & 168.0 & 166.0 & 1.31 & & & $\beta$ & 149.0 & 148.0 & 0.90 & 1.2 & 1.3 \\
\hline \multirow[t]{3}{*}{$\Gamma 10 h$} & {$[10 \overline{1} 0] \gamma$} & 17.2 & 14.0 & 0.28 & 0.36 & 1.3 & & 0.8 & & & & \\
\hline & {$[11 \overline{2} 0] \gamma$} & 17.3 & 14.1 & 0.27 & 0.31 & 1.2 & & 0.8 & & & & \\
\hline & {$[0001] \gamma$} & 9.4 & 8.0 & 0.20 & 0.25 & 1.3 & & 0.6 & & & & \\
\hline
\end{tabular}


TABLE V. Fermi-surface areas and cyclotron masses for Zr and Hf. The experimental results for $\mathrm{Zr}$ are taken from Ref. 19 .

\begin{tabular}{|c|c|c|c|c|c|c|c|}
\hline \multicolumn{8}{|c|}{ Zirconium } \\
\hline \multirow{2}{*}{\multicolumn{2}{|c|}{ Direction }} & \multicolumn{2}{|c|}{$\begin{array}{c}\text { Extremal } \\
\text { areas (MG) }\end{array}$} & \multicolumn{2}{|c|}{$\begin{array}{c}\text { Cyclotron } \\
\text { masses }\left(m / m_{0}\right)\end{array}$} & \multicolumn{2}{|c|}{ Hafnium } \\
\hline & & $\begin{array}{l}\text { areas } \\
\text { Theory }\end{array}$ & Expt. & $\begin{array}{l}\text { masses } \\
\text { Theory }\end{array}$ & $\begin{array}{l}\left.n / m_{0}\right) \\
\text { Expt. }\end{array}$ & $\begin{array}{c}\text { Extremal } \\
\text { areas (MG) }\end{array}$ & $\begin{array}{c}\text { Cyclotron } \\
\text { masses }\left(m / m_{0}\right)\end{array}$ \\
\hline \multirow[t]{5}{*}{$\Gamma A 3 h$} & {$[0001]$} & 27.9 & & 0.72 & & 29.3 & 0.56 \\
\hline & {$[0001] \alpha$} & 28.2 & 34.58 & 0.74 & 0.95 & 2.0 & 0.52 \\
\hline & {$[0001]$} & 3.3 & & 0.57 & & 2.1 & 0.55 \\
\hline & {$[0001]$} & 6.8 & & 0.58 & & 1.8 & 0.61 \\
\hline & {$[0001]$} & & & & & 13.9 & 0.94 \\
\hline \multirow[t]{4}{*}{$\Gamma A 4 h$} & [0001] & 40.8 & & 0.57 & & 39.7 & 0.79 \\
\hline & {$[0001] \beta$} & 31.0 & 38.00 & 0.89 & 1.14 & 51.4 & 1.08 \\
\hline & {$[0001] \gamma$} & 36.6 & 49.88 & 1.14 & 1.45 & 14.4 & 0.98 \\
\hline & {$[0001]$} & 7.0 & & 0.59 & & & \\
\hline \multirow[t]{4}{*}{$H 5 e$} & {$[10 \overline{10} 0]$} & 43.0 & & 2.54 & & 47.4 & 1.54 \\
\hline & {$[11 \overline{2} 0] \zeta$} & 43.5 & 41.25 & 2.39 & 1.83 & 47.6 & 1.43 \\
\hline & {$[0001]$} & 37.7 & & 2.24 & & 51.0 & 1.93 \\
\hline & [0001] & 54.3 & & 2.10 & & 53.3 & 1.18 \\
\hline \multirow[t]{3}{*}{$H 6 e$} & {$[10 \overline{1} 0]$} & 8.7 & & 2.34 & & 15.5 & 1.50 \\
\hline & {$[11 \overline{2} 0] \eta$} & 8.5 & 16.85 & 1.42 & & 15.9 & 1.28 \\
\hline & {$[0001]$} & 34.2 & & 2.41 & & 39.3 & 1.21 \\
\hline \multirow[t]{2}{*}{$H 56 e$} & {$[11 \overline{2} 0] \delta$} & 26.0 & 28.99 & 1.90 & 1.75 & & \\
\hline & {$[10 \overline{1} 0]$} & 25.8 & & 2.44 & & & \\
\hline
\end{tabular}

mentally observed areas. From among the various possibilities, the $\gamma$ orbit has been tentatively identified by its mass.

In analogy with $\mathrm{Ti},{ }^{27}$ the $\zeta, \eta$, and $\delta$ oscillations can be associated with orbits on the two $H$-centered surfaces. $\delta$ involves magnetic breakdown at the hexagonal face of the Brillouin zone, and its area is the mean of those of $\zeta$ and $\eta$, as is observed to a very good approximation experimentally. Further orbits involving magnetic breakdown near the line $H K$ are seen in $\mathrm{Ti}$, but the spin-orbit coupling is sufficiently large that they have not yet been observed in $\mathrm{Zr}$. The agreement between the theoretical and experimental $\eta$ areas is rather poor but, because of the flatness of the bands, relative shifts of only about $5 \mathrm{mRy}$ are required to bring them into coincidence. The calculated masses are surprisingly large, compared with the measured values, but these are again very sensitive to the precise positions of the bands.

The calculated Fermi surface of Hf, illustrated in Fig. 18, is very similar to that of $\mathrm{Zr}$ and, in this case, the $\Delta_{2}$ band approaches the Fermi level very closely. If our calculated $d$ band is indeed too low, as is indicated by the heat-capacity results, it is probable that the $\Gamma A 3 h$ surface is also disconnected.

\section{CONCLUSION}

In this paper, we have tested the linear-MTO method by comparing calculations based on it with detailed experimental results for some hep transition metals. The close agreement between experiment and theory for $\mathrm{Ru}$ and Os demonstrate, as did earlier comparisons with the RAPW method for $\mathrm{Yb}$, that it is a technique for solving the Schrödinger equation in a solid which gives energy eigenvalues with an uncertainty which is generally small compared with that inherent in present methods of constructing a priori potentials. The principal advantage of the method is that it is computationally extremely fast, so that it is well suited, for example, for calculations of more complex properties involving the electronic eigenfunctions, and for self-consistent calculations on complicated structures with many different atoms in the unit cell. In addition, the concepts of canonical bands and potential parameters are physically transparent and allow a simple understanding of the electronic structure. Furthermore, adjustments in the potential parameters may readily be made to improve the agreement with experimental data in the spirit of, but in a more straightforward and physically meaningful manner than, earlier interpolation schemes.

Together with the earlier calculations mentioned in the Introduction, our results demonstrate again the success of the muffin-tin potential constructed by superposing atomic charge densities, with the Slater $\rho^{1 / 3}$ approximation for exchange and correlation. In calculations on transition metals, such a potential gives the relative positions of the $s, p$, 
and $d$ bands to within about $10 \mathrm{mRy}$, and therefore a Fermi surface which is almost always qualitatively correct ( $\mathrm{Zr}$ is the only known exception) and is generally also in impressive quantitative agreement with experimental results. There has been some question of whether such a potential is suitable for transition metals with few $d$ electrons, or for the $3 d$ elements, but the success of the calculations on $^{27} \mathrm{Ti}$ appears to set such doubts at rest. The difficulties with such metals appear to be associated with the strong hybridization near the bottom of the $d$ band, which makes the Fermi surface very sensitive to the precise band positions, rather than with any inherent weakness in the potential.

It is of great importance for the further development of our understanding of transition metals that such a standard potential is available, even though the theoretical justification for its sucess is far from complete. The standard potential is not selfconsistent, but provides a prescription for a local one-electron potential which is better than that resulting from almost all more sophisticated selfconsistent procedures. It is a major challenge to construct and justify a self-consistent potential which gives results in as good agreement with experiment as the standard potential, and such a development is essential for further progress in understanding transition-metal compounds.

Our discussion suggests possibilities for further studies of the metals which we have considered. $\mathrm{Ru}$ is the best understood of them, and measurements of its galvanomagnetic properties would clearly be of interest, especially because the $K M 8 h$ surface supports two independent sets of open orbits, one of which is destroyed by magnetic breakdown. A more precise measurement of the electronic heat capacity would also be of interest. We have argued that two sets of very small hole surfaces may well exist in Os, and a further search for dHvA oscillations associated with them seems worthwhile. If further results become available on the small sections of Fermi surface in Os, a recalculation of the corresponding areas using a much finer mesh would be justified, since our results for these surfaces are at present only semiquantitative. The experiments on both $\mathrm{Ru}$ and $\mathrm{Os}$ are sufficiently precise and detailed that a fitting of the Fermi surface areas using the $s, p$, and $d$ logarithmic derivatives or phase shifts (with spinorbit coupling) as adjustable parameters would be useful. This would give further information about the validity of the muffin-tin approximation and the standard potential.

The experimental data available on $\mathrm{Zr}$ are much less complete, but our interpretation of them appears plausible, especially in view of the successful explanation of the very similar properties of Ti. It might be worthwhile to adjust the potential parameters empirically, in order to obtain a better agreement with the experimental results, but further dHvA measurements are probably necessary before the band structure can be specified with high precision. The difficulties of purifying Hf provide a formidable obstacle to obtaining information about its Fermi surface, but it might be possible to prepare a sample of sufficiently high purity to determine whether there are open orbits in the [0001] direction, even in very high magnetic fields, in which magnetic breakdown is complete.

Although our quantitative knowledge of the electronic structure of the hcp transition metals is not generally as precise as that of the cubic metals, and relatively little work has yet been done on examining the energy levels away from the Fermi level by optical and photoemission techniques, it appears that we now have a good physical understanding of their energy bands and can calculate many electronic properties with satisfactory accuracy. A natural extension of this work would be an attempt to attain a similar understanding of closely-packed transition-metal compounds, for which, at present, there is a paucity of both reliable calculations and detailed experimental results.

\section{ACKNOWLEDGMENTS}

Part of the research reported in this paper was performed while A.R. M. was a guest scientist for a summer at the Rockwell Science Center. It is a pleasure to thank the director, Dr. T. L. Loucks, for the hospitality of the laboratory and for many fruitful discussions. Helpful comments from Dr. D. G. Pettifor are also gratefully acknowledged.
${ }^{1}$ T. L. Loucks, Phys. Rev. 144, 504 (1966).

${ }^{2} \mathrm{~T}$. L. Loucks, Phys. Rev. $\underline{159}, 544$ (1967).

${ }^{3}$ S. C. Keeton and T. L. Loucks, Phys. Rev. 168, 672 (1968).

${ }^{4}$ L. F. Mattheiss, Phys. Rev . 151, 450 (1966).

${ }^{5}$ A. S. Joseph, A. C. Thorsen, and L. E. Valby, Phys. Rev. 150, 523 (1966).

${ }^{6}$ W. A. Reed, E. Fawcett, and R. R. Soden, Phys. Rev. 139, A1557 (1965).

${ }^{7}$ L. R. Testardi and R. R. Soden, Phys. Rev. 158,581
(1967).

${ }^{8}$ L. F. Mattheiss, Phys. Rev. $\underline{133}$, A1399 (1964).

${ }^{9}$ J. C. Slater, Phys. Rev. $81, \overline{385}$ (1951).

${ }^{10}$ T. L. Loucks, Phys. Rev. 139 , A1181 (1965); L. F. Mattheiss, Phys. Rev. B 1,373 (1970); N. E. Christensen and B. Feuerbacher, ibid. 10, 2349 (1974).

${ }^{11}$ O. K. Andersen, Phys. Rev. B 2,883 (1970).

${ }^{12}$ N. E. Christensen, Phys. Rev. B 4,3321 (1971).

${ }^{13}$ S. L. Altmann and J. C. Bradley, Proc. Phys. Soc. Lond. 92,764 (1967). 
${ }^{14}$ O. K. Andersen, Solid State Commun. 13, 133 (1973).

${ }^{15} \mathrm{O}$. K. Andersen, preceding paper, Phys. Rev. B 12 , 3060 (1975).

${ }^{16}$ P. T. Coleridge, J. Low Temp. Phys. 1,577 (1969).

${ }^{17}$ G. N. Kamm and J. R. Anderson, Phys. Rev. B 2 , 2944 (1970).

${ }^{18} \mathrm{O}$. Jepsen and O. K. Andersen (unpublished).

${ }^{19}$ P. M. Everett, Phys. Rev. B $\underline{6}, 3553$ (1972); $\underline{6}, 3559$ (1972).

${ }^{20} \mathrm{O} . \mathrm{K}$. Andersen and O. Jepsen (unpublished).

${ }^{21}$ O. Jepsen and O. K. Andersen, Solid State Commun. $\underline{9}$, 1763 (1971).
${ }^{22}$ W. B. Pearson, A Handbook of Lattice Spacings and Structures of Metals and Alloys (Pergamon, New York, 1958).

${ }^{23} \mathrm{~F}$. Heiniger, E. Bucher, and J. Muller, Phys. Kondens. Mater. $\underline{5}, 243$ (1966).

${ }^{24}$ W. L. McMillan, Phys. Rev. 167, 331 (1968).

${ }^{25}$ C. R. Brown, J. P. Kalejs, F. D. Manchester, and J. M. Perz, Phys. Rev. B $\underline{6}, 4458$ (1972).

${ }^{26}$ G. N. Kamm and J. R. Anderson, in Low Temperature Physics-LT13 (Plenum, New York, 1974), Vol. 4.

${ }^{27}$ O. Jepsen, Phys. Rev. B 12 , 2988 (1975). 